\title{
Modern Semi-Active Control Schemes for a Suspension with MR Actuator for Vibration Attenuation ${ }^{\dagger}$
}

\author{
Kevin Herubiel Floreán-Aquino ${ }^{1}$, Manuel Arias-Montiel ${ }^{2, * \mathbb{D}}$, Jesús Linares-Flores ${ }^{2} \mathbb{D}$, \\ José Gabriel Mendoza-Larios ${ }^{3}$ and Álvaro Cabrera-Amado ${ }^{4}$
}

1 Division of Posgraduate Studies, Technological University of the Mixteca, Huajuapan de León 69000, Oaxaca, Mexico; floreankh@gmail.com

2 Institute of Electronics and Mechatronics, Technological University of the Mixteca, Huajuapan de León 69000, Oaxaca, Mexico; jlinares@mixteco.utm.mx

3 Institute of Industrial and Automotive Engineering, Technological University of the Mixteca, Huajuapan de León 69000, Oaxaca, Mexico; jgml@mixteco.utm.mx

4 Department of Mechatronics Engineering, Papaloapan University, Loma Bonita 68400, Oaxaca, Mexico; alvaroca_1@hotmail.com

* Correspondence: mam@mixteco.utm.mx; Tel.: +52-9535320214

$+\quad$ This paper is an extended version of our paper published in Florean-Aquino, K.H.; Arias-Montiel, M.; Lugo-Gonzalez, E.; Cabrera-Amado, A. Single and Multiple Positive Position Feedback Control of a Magnetorheological Automotive Suspension. In Proceedings of the National Congress on Automatic Control, Puebla, Mexico, 23-25 October 2019.

check for

updates

Citation: Floreán-Aquino, K.H.; Arias-Montiel, M.; Linares-Flores, J.; Mendoza-Larios, J.G.;

Cabrera-Amado, Á. Modern

Semi-Active Control Schemes for a Suspension with MR Actuator for

Vibration Attenuation. Actuators 2021, 10, 22. https://doi.org/10.3390/ act10020022

Academic Editor: Norman M. Wereley

Received: 29 December 2020

Accepted: 22 January 2021

Published: 27 January 2021

Publisher's Note: MDPI stays neutral with regard to jurisdictional clai$\mathrm{ms}$ in published maps and institutional affiliations.

Copyright: $\odot 2021$ by the authors. Licensee MDPI, Basel, Switzerland. This article is an open access article distributed under the terms and conditions of the Creative Commons Attribution (CC BY) license (https:// creativecommons.org/licenses/by/ $4.0 /)$.

\begin{abstract}
This article describes semi-active modern control schemes for a quarter-vehicle suspension with a magnetorheological damper (MRD) to attenuate vibrations and simultaneously improve the passenger comfort and the vehicle road-holding. The first solution is a multiple positive position feedback (MPPF) control scheme to attenuate the vibration amplitude at the two modal frequencies. The second solution is based on elementary passivity considerations on the exact regulation error dynamics passive output. The passive output feedback is used to improve the control aims. Finally, the third solution deals with a disturbance rejection control (DRC) based on an extended state observer. The three proposed control schemes consider an inverse polynomial model of a commercial MRD for numerical implementation and are evaluated by comfort and road-holding performance indexes proposed in the literature. Furthermore, the effects of variation in the sprung mass (emulating different number of passengers) on the controllers' performance is analysed. The numerical results show in both scenarios (constant and variable sprung mass) that passivity based control (PBC) and DRC improve the performance indexes compared with the classical sky-hook control and the open-loop systems with a different constant current input for the MRD. Obtained results for damping force and power consumption are within the operation range of the considered commercial MRD showing the viability for experimental implementation of the proposed control schemes.
\end{abstract}

Keywords: magnetorheological damper; differential flatness; extended state observer; passivitybased control

\section{Introduction}

Rheological actuators (RA) are the most promising semi-active devices in energy dissipation of structures under dynamic loads. These actuators contain a fluid which is able to modify its rheological structure (yield stress and apparent viscosity) through the action of an electrical field (electrorheological) or a magnetic field (magnetorheological) [1] According to [2], RA are passive-dissipative devices because they do not provide energy to the controlled system. Moreover, the low power consumption, large force capacity, high bandwidth, low cost, force controllability and quick reaction time make magnetorheological dampers (MRD) a viable option for vibration control systems in civil engineering applications, impact absorption and vibration isolation technology in industrial engineering and 
heavy vehicles, as well as advanced prosthetics in biomedical fields [3-5]. In automotive applications, MRD are used in the development of electronic suspension (ES) systems in order to reduce the transmissibility of mechanical vibration caused by unknown road irregularities improving passenger comfort (mainly related to vibration transmitted to passengers from road irregularities, in this work measured through the chassis displacement) as well as vehicle road-holding (related to the tire deflection, measured by the difference between the tire displacement and movement caused by the road profile) [6,7]. The automotive ES based on magnetorheological dampers (MRD) have demonstrated excellent real-time performance. In addition, they present other relevant characteristics such as response delays in the milliseconds order and low power consumption [6-8]. In the classification of controllable suspension systems presented by Savaresi et al. [7], typical ranges of power consumption for different types of electronically controlled suspension are provided. According to this information, semi-active suspension systems, mainly based on RA have a power request from a few tens of Watts, while the fully active suspensions power request is around tens of kilowatts. The control input of a MRD is an electrical current from a coil which induces a magnetic field on the magnetorheological fluid (MRF). Regarding MDR characterization, parametric models were developed to describe the hysteretic behaviour of the force-velocity curve [4,9]. Furthermore, there are non-parametric models to describe the hysteretic loop by polynomial velocity functions. This type of model allows an expression of the electric current as a function of the desired damping force to semi-active control (SAC) applications [10]. In this case, the performance of suspensions with MRD strongly depends on the intrinsic characteristics of the SAC scheme. Classic SAC strategies are based on discontinuous switching according to two different performance approaches: comfort or road-holding. Some examples of these approaches are Ground-hook (road-holding), Acceleration Driven Damper (comfort), sky-hook (comfort) and SH-ADD (comfort and road-holding) [7]. The classic SAC presents some drawbacks such as chattering to high frequencies which could excite non-modelled dynamics in mechanical systems and a reduced bandwidth which limits the capacity for improving passenger comfort and road-holding simultaneously $[6,11]$. As an alternative for overcoming the limitations of classic SAC, modern SAC schemes have emerged mainly based on Linear Parameter Varying (LPV) approach [8], optimal control theories [12], robust control [13,14], adaptive control [15], modal and multi-modal control $[16,17]$ and fault tolerant control [18].

In the model-based control schemes, the unknown and unmeasurable external disturbances highly affect the closed-loop system performance. In this regard, extended-state observers (ESO) have been proposed in the active disturbance rejection control (ADRC) approach [19] to estimate unmeasurable states and unknown disturbances as well as to provide adaptability, and robustness to the controller. Recently, ESO have found applications in uncertain mechanical systems with various control schemes [20-23].

According to the consulted literature, one of the main drawbacks of classic SAC schemes is their inability to improve passenger comfort and vehicle road-holding simultaneously. Moreover, some modern SAC schemes have proved effective in the main vibration mode only [13-16,18], and just a few works take into account the MR damper dynamics $[8,15]$. Moreover, the effects of the system parameters variation on the controller performance is rarely considered, even in the robust control schemes. This work is an extended and improved version of the results reported in [17] and presents the design of three modern control schemes for a quarter-vehicle suspension with MRD taking into account the actuator dynamics from an inverse polynomial model previously characterized. Furthermore, the control schemes are numerically evaluated by comfort and road-holding indexes, while changes in the sprung mass (emulating different number of passengers) are considered in the controllers' performance. The methodology used in this article is presented in Figure 1. The research development is divided into four stages: modeling, covering the suspension dynamics as well as the polynomial model to characterize the MRD; control design, where the theoretical background to synthesise the proposed SAC schemes is given; simulation, where the proposed SAC schemes are numerically proved taking into 
account variations in the sprung mass value; and evaluation through performance indexes for comfort and road-holding. The principal aim of the proposed controllers is to achieve a high comfort performance for the passenger as well as obtaining ride stability (road holding). The first solution was taken from [17] and uses a modal coordinate form to extend and control the primary system dynamic by virtual filters tuned to the principal modal frequencies. Another solution based on elementary passivity considerations on the exact regulation error dynamics passive output is presented using a hamiltonian form to improve both control aims with low power consumption. Finally, a robust adaptive controller based on differential flatness is developed where an ESO is incorporated to add robustness and adaptability to the primary control system. All these proposed schemes are numerically implemented by considering an inverse polynomial model of a commercial MRD and evaluated by performance indexes reported in the literature. The article is organized according to the activities presented in the methodology as follows: Section 2 presents the vertical model of a quarter car and an inverse polynomial model for a commercial magnetorheological damper experimentally characterized in [24]. The following sections presents the main results related to: the design of two modal controllers (Section 3); the design of a passivity-based controller (PBC) (Section 4); and the development of a robust and adaptive controller using the system flatness property (Section 5). Section 6 briefly describes the performance indexes used to evaluate the proposed control schemes and shows the numerical results from simulation tests of a closed-loop semi-active suspension system. Finally, Section 7 presents the conclusions of this work and some suggestions for further work.

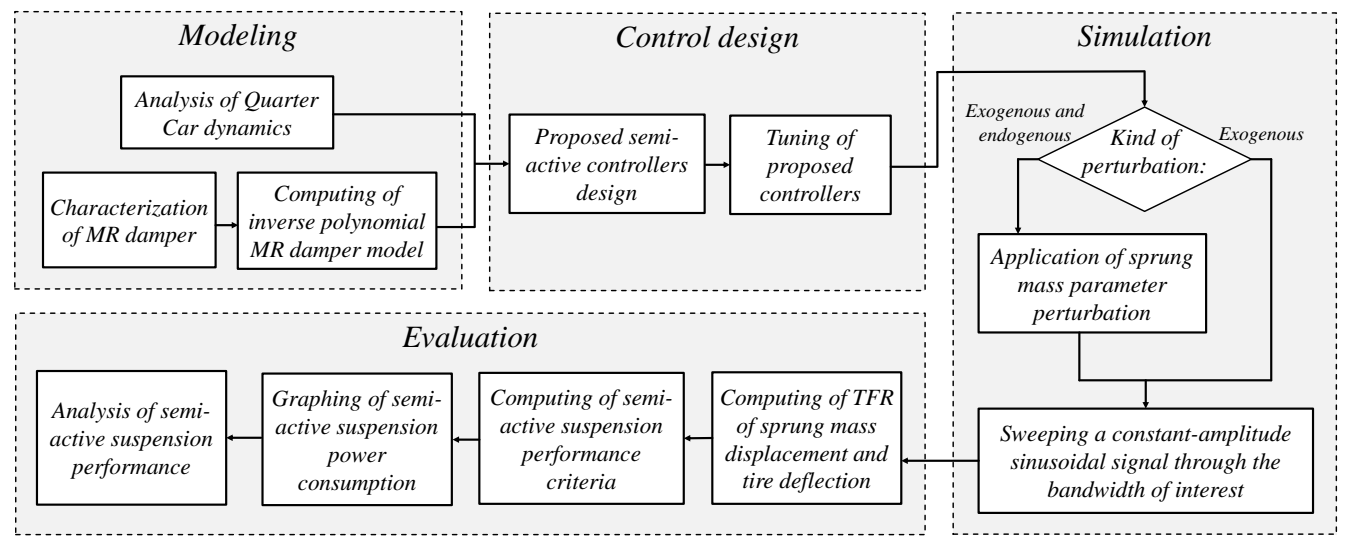

Figure 1. Proposed research methodology.

\section{Modeling of a Quarter Automotive Suspension and MRD Dynamics}

\subsection{Vertical Model of a Quarter Car (VMQC)}

The vertical model of a quarter car (VMQC) defines the dynamic relationships of a sprung mass $m_{s}$ (the portion of the total mass of the vehicle that is supported by the suspension) and an unsprung mass $m_{u}$ (the mass of the suspension, wheels and other components directly connected to them), see Figure 2.

The suspension system consists of a linear stiffness $k_{s}$ and an apparent damping coefficient $c_{s}$. The tire stiffness is assumed as linear with spring constant $k_{u}$ and the tire damping is neglected. The disturbance caused by the road profile $z_{r}(t)$ is unknown but is perfectly bounded. The semi-active MRD force $f_{a}$ is the control input which is a function of the electric current $i_{a}$ between the MRD terminals. The motion equations for the system masses can be obtained by applying the second law of Newton as

$$
\begin{gathered}
m_{s} \ddot{z}_{s}+c_{s}\left[\dot{z}_{s}-\dot{z}_{u}\right]+k_{s}\left[z_{s}-z_{u}\right]+f_{a}\left(i_{a}\right)=0 \\
m_{u} \ddot{z_{u}}+k_{u}\left[z_{u}-z_{r}\right]=c_{s}\left[\dot{z}_{s}-\dot{z}_{u}\right]+k_{s}\left[z_{s}-z_{u}\right]+f_{a}\left(i_{a}\right)
\end{gathered}
$$

where $\ddot{z}_{s}, \dot{z}_{s}, z_{s}$ are the acceleration, velocity and displacement of $m_{s}$ respectively, and $\ddot{z}_{u}, \dot{z}_{u}$, $z_{u}$ are the acceleration, velocity and displacement of $m_{u}$ respectively. Moreover, there is a 
set of strictly positive real constants $\left\{m_{\text {min }}, m_{\max }, i_{\text {lim }}, f_{o}, r_{\text {max }}\right\}$ such as $m_{\text {min }} \leq m_{s} \leq m_{\text {max }}$, $0 \leq i_{a} \leq i_{\text {lim }},\left|f_{a}\right| \leq f_{o}$, and $\left|z_{r}\right| \leq r_{\text {max }} . m_{\text {min }}, m_{\text {max }}$ define the range of the sprung mass variation, $i_{\text {lim }}$ is the maximum input current permissible for MRD, and $f_{o}$ is the maximum damping force provided by MRD, $r_{\max }$ is a positive number that represents the upper limit of the disturbance amplitude indicating this variable is bounded.

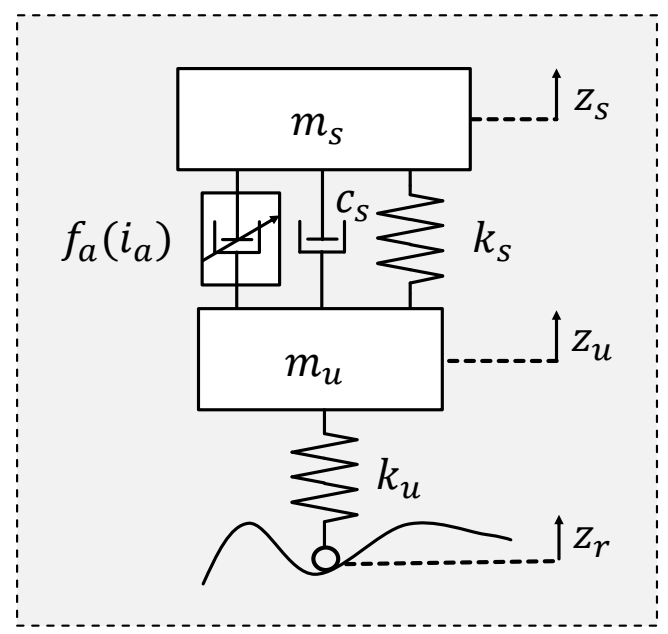

Figure 2. Vertical model of a quarter car with semi-active suspension [7].

\subsection{MRD Dynamics}

This work considers a polynomial model for the MRD. The polynomial model parameters can be experimentally estimated from force and displacement measurements only, and this model is an expression of the damping force as a function of piston velocity and the input current of the damper. The order of the polynomial can be adjusted to the experimental data while its coefficients can be determined by curve fitting. In addition, an inverse model to obtain the input current to achieve a desirable damping force can be easily obtained which is very useful for implementing SAC schemes [24].

The damping force of the MRD can be expressed by [10]

$$
f_{a}=\sum_{i=0}^{n} a_{i} \dot{z}_{\text {def }}^{i}, \quad n=\text { polynomial degree }
$$

where $\dot{z}_{\text {def }}=\dot{z}_{s}-\dot{z}_{u}$ is the piston velocity and $a_{i}$ is the experimental coefficient that can be represented as a function of the intensity of the input current in a linear form as

$$
a_{i}=b_{i}+c_{i} i_{a}, \quad i=0,1, \ldots, n
$$

where $b_{i}$ and $c_{i}$ are constant to be determined from curve fitting.

Combining Equations (2) and (3), the damping force can be expressed by

$$
f_{a}=\sum_{i=0}^{n}\left(b_{i}+c_{i} i_{a}\right) \dot{z}_{d e f}^{i}
$$

In a SAC problem a desired damping force $f_{a}$ is calculated to attenuate the vibration amplitudes in the suspension system. This desired force can be used to determine the input current needed in the MRD to achieve this force. For the polynomial model, the control input is determined from Equation (4) as

$$
i_{a}=\frac{f_{a}-\sum_{i=0}^{n}\left(b_{i} \dot{z}_{d e f}^{i}\right)}{\sum_{i=0}^{n}\left(c_{i} \dot{z}_{\text {def }}^{i}\right)}
$$

The commercial MRD RD-8040-1 by LORD Corporation ${ }^{\circledR}$ experimentally characterized in [24] is considered in this work to implement the proposed SAC schemes. For this 
MRD, the inverse polynomial (IP) model is defined by (6) with the numerical parameters presented in Table 1 [17].

$$
i_{a}\left(f_{a}\right)=\left\{\begin{array}{lc}
\frac{f_{a}-b_{2}^{+} \dot{z}_{d e f}^{2}-b_{1}^{+} \dot{z}_{d e f}-b_{0}^{+}}{c_{2}^{+} \dot{z}_{d e f}^{2}+c_{1}^{+} \dot{z}_{d e f}+c_{0}^{+}} & \ddot{z}_{d e f}>0 \\
\frac{f_{a}-b_{2}^{-} \dot{z}_{d e f}^{2}-b_{1}^{-} \dot{z}_{d e f}-b_{0}^{-}}{c_{2}^{-} \dot{z}_{d e f}^{2}+c_{1}^{-} \dot{z}_{d e f}+c_{0}^{-}} & \ddot{z}_{\text {def }}<0
\end{array}\right\}
$$

Table 1. Inverse polynomial model parameters for positive and negative acceleration of the suspension deflection (copyright AMCA (2019), reproduced from [17]).

\begin{tabular}{ccccc}
\hline \multicolumn{3}{c}{$\ddot{z}_{\text {def }}>\mathbf{0}$} & \multicolumn{2}{c}{$\ddot{z}_{\text {def }}<\mathbf{0}$} \\
\hline$j$ & $\boldsymbol{b}_{j}^{+}$ & $\boldsymbol{c}_{j}^{+}$ & $\boldsymbol{b}_{j}^{-}$ & $\boldsymbol{c}_{j}^{-}$ \\
\hline 0 & 3.2679 & 224.8581 & -115.2069 & -259.8339 \\
1 & 7.9904 & 50.1593 & 8.2080 & 51.2396 \\
2 & -0.0836 & -0.7803 & 0.2687 & 0.7833 \\
\hline
\end{tabular}

\section{Modal and Multimodal Controller Design}

The development presented in this section is based on the results previously presented in [17]. The aim of modal control is to provide damping to certain modes of the system, which dominate the dynamic response. Positive Position Feedback (PPF) [25] is a modal control method, which provides additional dynamics to the system through the control law. Both PPF and its variant Multi Positive Position Feedback (MPPF) [26,27] are analysed in this work to provide comfort and performance on the road (road-holding) of the VMQC. These objectives are related with two main modes of the system (the first in low frequencies, the second in high frequencies). A modal control approach refers to the procedure of decomposing the dynamic equations of a structure into modal coordinates and designing the control law in this modal coordinate system [28]. Note that (1) can be expressed by a modal coordinate form as follows:

$$
M \ddot{z}+C \dot{\vec{z}}+K \vec{z}=\vec{B}_{f} u+\vec{B}_{r} z_{r}
$$

where the vertical displacement masses vector is defined as $\vec{z}=\left[\begin{array}{ll}z_{s} & z_{u}\end{array}\right]^{T}$ and the control input as $u=f_{a}$; here the matrices $M, K$ and $C$ are symmetric and positive definite as

$$
M=\left[\begin{array}{cc}
m_{s} & 0 \\
0 & m_{u}
\end{array}\right], \quad C=\left[\begin{array}{cc}
c_{s} & -c_{s} \\
-c_{s} & c_{s}
\end{array}\right], \quad K=\left[\begin{array}{cc}
k_{s} & -k_{s} \\
-k_{s} & k_{s}+k_{u}
\end{array}\right]
$$

the vectors $\vec{B}_{f}, \vec{B}_{r}$ are defined by

$$
\vec{B}_{f}=\left[\begin{array}{c}
-1 \\
1
\end{array}\right] \quad \vec{B}_{r}=\left[\begin{array}{c}
0 \\
k_{u}
\end{array}\right]
$$

\subsection{Positive Position Feedback (PPF) Controller Design}

The positive position terminology comes from the fact that the position coordinate of the structure equation is positively fed to a virtual second-order filter, and the position coordinate of the compensator equation is positively feedback to the structure [28].

The PPF control is implemented by using an auxiliary dynamic system (virtual compensator) which can be defined by

$$
\ddot{\eta}+2 \zeta_{f} \omega_{f} \dot{\eta}+\omega_{f}^{2} \eta=g \omega_{f}^{2} \vec{B}_{f}^{T} \vec{z}
$$




$$
u=g \omega_{f}^{2} \eta
$$

where $\eta$ is the generalized coordinate of the virtual compensator (secondary system), $\zeta_{f}, \omega_{f}>0$ are the damping ratio and natural frequency of the controller, and $g$ is a positive constant. These control parameters can be chosen so that the response has the desired damping. Coupling the system dynamics in (7) with the PPF controller in (10), which assumes no external force, yields

$$
\left[\begin{array}{cc}
M & 0 \\
0 & 1
\end{array}\right]\left[\begin{array}{c}
\ddot{\vec{z}} \\
\ddot{\eta}
\end{array}\right]+\left[\begin{array}{cc}
C & 0 \\
0 & 2 \zeta_{f} \omega_{f}
\end{array}\right]\left[\begin{array}{c}
\dot{\vec{z}} \\
\dot{\eta}
\end{array}\right]+\left[\begin{array}{cc}
K & -g \omega_{f}^{2} \vec{B}_{f} \\
-g \omega_{f}^{2} \vec{B}_{f}^{T} & \omega_{f}^{2}
\end{array}\right]\left[\begin{array}{l}
\vec{z} \\
\eta
\end{array}\right]=\left[\begin{array}{l}
0 \\
0
\end{array}\right]
$$

Since the matrices $M, C$, and $K$ in (7) are symmetric and positive definite, both the augmented damping matrix and the augmented mass matrix are also symmetric and positive definite, so the closed-loop stability will depend on the definiteness of the augmented stiffness matrix.

Closed-Loop Stability

The augmented stiffness matrix in (12) is given by

$$
\hat{K}=\left[\begin{array}{cc}
K & -g \omega_{f}^{2} \vec{B}_{f} \\
-g \omega_{f}^{2} \vec{B}_{f}^{T} & \omega_{f}^{2}
\end{array}\right]
$$

which will be positive definite if for whichever test vector $\vec{q} \in \Re^{n}$ it is satisfied that

$$
\vec{q}^{T} \hat{K} \vec{q}>0
$$

Considering the test vector as

$$
\vec{q}^{T}=\left[\begin{array}{ll}
\vec{q}_{1}^{T} & \vec{q}_{2}^{T}
\end{array}\right]^{T}
$$

where $\vec{q}_{1}, \vec{q}_{2} \in \Re^{n}$, substitution of (15) in the Inequality (14) can be expressed as

$$
\begin{gathered}
\vec{q}^{T} \hat{K} \vec{q}=\left[\begin{array}{ll}
\vec{q}_{1}^{T} & \vec{q}_{2}^{T}
\end{array}\right]\left[\begin{array}{cc}
K & -g \omega_{f}^{2} \vec{B}_{f} \\
-g \omega_{f}^{2} \vec{B}_{f}^{T} & \omega_{f}^{2}
\end{array}\right]\left[\begin{array}{l}
\vec{q}_{1} \\
\vec{q}_{2}
\end{array}\right] \\
\vec{q}^{T} \hat{K} \vec{q}=\vec{q}_{1}^{T} K \vec{q}_{1}-g \omega_{f}^{2} \vec{q}_{1}^{T} \vec{B}_{f} \vec{q}_{2}-g \omega_{f}^{2} \vec{q}_{2}^{T} \vec{B}_{f}^{T} \vec{q}_{1}+\omega_{f}^{2} \vec{q}_{2}^{T} \vec{q}_{2}
\end{gathered}
$$

Completing the square and factoring in (17) yields

$$
\vec{q}^{T} \hat{K} \vec{q}=\vec{q}_{1}^{T}\left(K-g^{2} \omega_{f}^{2} B_{f}^{T} B_{f}\right) \vec{q}_{1}+\left(g \omega_{f} B_{f}^{T} \vec{q}_{1}-\omega_{f} \vec{q}_{2}\right)^{T}\left(g \omega_{f} B_{f}^{T} \vec{q}_{1}-\omega_{f} \vec{q}_{2}\right)
$$

Since the second term in the right side of (18) is always non-negative, the augmented stiffness matrix $\hat{K}$ will be positive definite if the term $\left(K-g^{2} \omega_{f}^{2} B_{f}^{T} B_{f}\right)$ is a positive definite matrix.

Remark 1. The closed-loop system is asymptotically stable if the gain $g$ and the natural frequency of the virtual compensator $\omega_{f}$ are chosen such that $\left(K-g^{2} \omega_{f}^{2} B_{f}^{T} B_{f}\right)$ is positive definite.

\subsection{Multiple Positive Position Feedback (MPPF) Controller Design}

Multiple Positive Position Feedback (MPPF) is a modal control strategy to attenuate mechanical vibrations on different modes, which is an extension of the PPF approach. This scheme adds multiple virtual passive absorbers located in parallel form to the primary system. Non-disturbed suspension system in closed loop is given by

$$
M \ddot{\vec{z}}+C \dot{\vec{z}}+K \vec{z}=\vec{B}_{f} u
$$


where $M, C, K \in \Re^{2 x 2}, \vec{B}_{f} \in \Re^{2}, \vec{z} \in \Re^{2}$ and $u \in \Re$.

In analogy to the PPF approach, the auxiliary compensator in MPPF control is defined by two virtual second-order filters to simultaneously attenuate two modes, as follows

$$
\begin{aligned}
& \ddot{\eta}_{1}+2 \zeta_{1} \omega_{1} \dot{\eta}_{1}+\omega_{1}^{2} \eta_{1}=g_{1} \omega_{1}^{2} \vec{B}_{f}^{T} \vec{z} \\
& \ddot{\eta}_{2}+2 \zeta_{2} \omega_{2} \dot{\eta}_{2}+\omega_{2}^{2} \eta_{2}=g_{2} \omega_{2}^{2} \vec{B}_{f}^{T} \vec{z}
\end{aligned}
$$

likewise, the input control is calculated by

$$
u=\sum_{i=1}^{2} g_{i} \omega_{i}^{2} \eta_{i} \quad g_{i}, \omega_{i} \in \Re
$$

where $\zeta_{i}$ and $\omega_{i}$ is the damping ratio and natural frequency of the $i$-th filter respectively. The virtual second-order filters' dynamics can be rewritten in a matrix form as follows

$$
I \ddot{\vec{\eta}}+2 \Gamma \Omega \dot{\vec{\eta}}+\Omega^{2} \vec{\eta}=G \Omega^{2} B^{T} \vec{z}
$$

Thus, the control law (22) becomes

$$
u=\vec{h} G \Omega^{2} \vec{\eta}
$$

here, $\vec{\eta}=\left[\begin{array}{ll}\eta_{1} & \eta_{2}\end{array}\right]^{T}$, and $I, \Gamma, \Omega, G \in \Re^{2 \times 2}$ are positive definite, $B \in \Re^{2 x 2}, \vec{h} \in \Re^{1 \times 2}$, and are given by

$$
I=\left[\begin{array}{ll}
1 & 0 \\
0 & 1
\end{array}\right], \Gamma=\left[\begin{array}{cc}
\zeta_{1} & 0 \\
0 & \zeta_{2}
\end{array}\right], \Omega=\left[\begin{array}{cc}
\omega_{1} & 0 \\
0 & \omega_{2}
\end{array}\right], G=\left[\begin{array}{cc}
g_{1} & 0 \\
0 & g_{2}
\end{array}\right] B=\left[\begin{array}{ll}
B_{f} & B_{f}
\end{array}\right], \vec{h}=\left[\begin{array}{ll}
1 & 1
\end{array}\right]
$$

Coupling the system dynamic in (7) with the MPPF controller in (25), and assuming no external force, yields

$$
\left[\begin{array}{cr}
M & 0 \\
0 & I
\end{array}\right]\left[\begin{array}{c}
\ddot{\vec{z}} \\
\ddot{\vec{\eta}}
\end{array}\right]+\left[\begin{array}{cc}
C & 0 \\
0 & 2 \Gamma \Omega
\end{array}\right]\left[\begin{array}{c}
\dot{\vec{z}} \\
\dot{\vec{\eta}}
\end{array}\right]+\left[\begin{array}{cc}
K & -B G \Omega^{2} \\
-G \Omega^{2} B^{T} & \Omega^{2}
\end{array}\right]\left[\begin{array}{l}
\vec{z} \\
\vec{\eta}
\end{array}\right]=\left[\begin{array}{l}
0 \\
0
\end{array}\right]
$$

Note that the matrix $B$ is equivalent to the matrix product of $B_{f}$ and the vector $\vec{h}=\left[\begin{array}{ll}1 & 1\end{array}\right]$. This form allows the closed-loop stability to be analyzed directly.

\section{Closed-Loop Stability}

In analogy to the PPF approach, the closed-loop stability will depend on the definiteness of the augmented stiffness matrix, which is expressed by

$$
\hat{K}=\left[\begin{array}{cc}
K & -B G \Omega^{2} \\
-G \Omega^{2} B^{T} & \Omega^{2}
\end{array}\right]
$$

and will be positive definite for whichever test vector $\vec{q} \in \Re^{n}$ is satisfied that

$$
\vec{q}^{T} \hat{K} \vec{q}>0
$$

Here, $\vec{q}$ is considered as

$$
\vec{q}^{T}=\left[\begin{array}{ll}
\vec{q}_{1}^{T} & \vec{q}_{2}^{T}
\end{array}\right]^{T}
$$

where $q_{1}, q_{2} \in \Re^{n}$. Thus, the left side of the inequality in (28) can be expressed as

$$
\begin{gathered}
\vec{q}^{T} \hat{K} \vec{q}=\left[\begin{array}{ll}
\vec{q}_{1}^{T} & \vec{q}_{2}^{T}
\end{array}\right]\left[\begin{array}{cc}
K & -B G \Omega^{2} \\
-G \Omega^{2} B^{T} & \Omega^{2}
\end{array}\right]\left[\begin{array}{l}
\vec{q}_{1} \\
\vec{q}_{2}
\end{array}\right] \\
\vec{q}^{T} \hat{K} \vec{q}=\vec{q}_{1}^{T} K \vec{q}_{1}-\vec{q}_{1}^{T} B G \Omega^{2} \vec{q}_{2}-\vec{q}_{2}^{T} G \Omega^{2} B^{T} \vec{q}_{1}+\vec{q}_{2}^{T} \Omega^{2} \vec{q}_{2}
\end{gathered}
$$


Completing the square and factoring in (31) yields

$$
\vec{q}^{T} \hat{K} \vec{q}=\vec{q}_{1}^{T}\left(K-G^{2} \Omega^{2} B^{T} B\right) \vec{q}_{1}+\left(G \Omega B^{T} \vec{q}_{1}-\Omega \vec{q}_{2}\right)^{T}\left(G \Omega B^{T} \vec{q}_{1}-\Omega \vec{q}_{2}\right)
$$

Remark 2. The closed-loop system will be asymptotically stable if $G$ and $\Omega$ are chosen such that $\left(K-G^{2} \Omega^{2} B^{T} B\right)$ is positive definite.

The proposed multimodal controller is illustrated in Figure 3 [17].

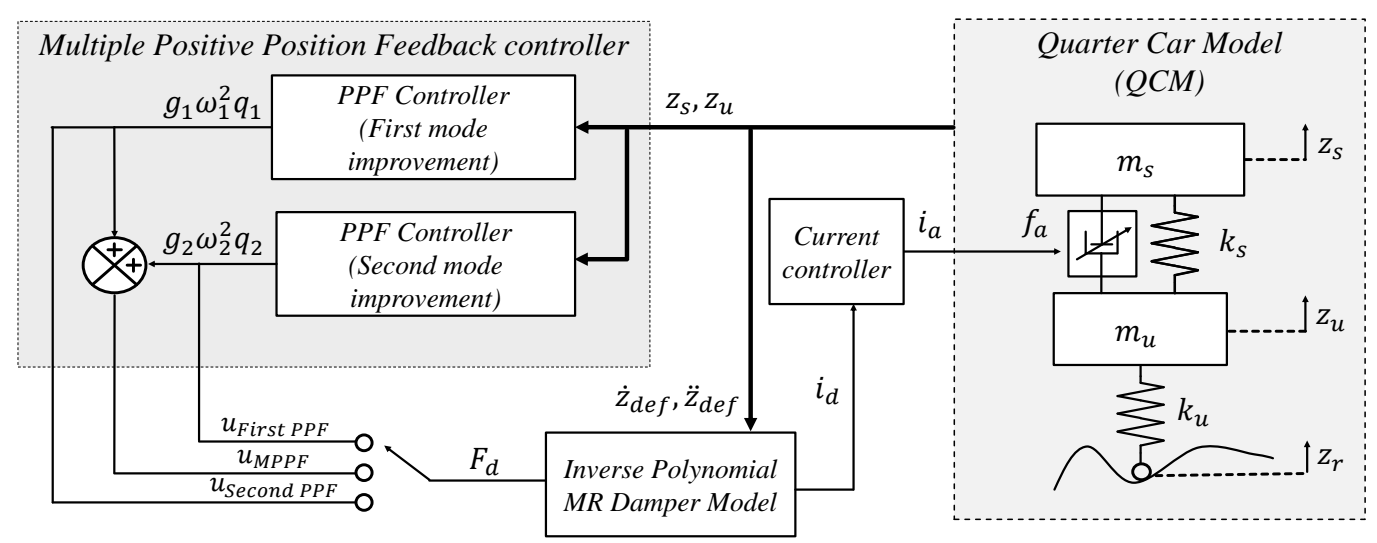

Figure 3. Proposed modal control system for semi-active suspension with magnetorheological damper (MRD) (copyright AMCA (2019), reproduced from [17]).

\section{Passivity-Based Controller (PBC) Design}

Passive systems are a class of dynamical systems which cannot store more energy than is supplied from external sources. This property is sufficient for solving regulation tasks in mechanical systems using the the passivity-based control (PBC) approach by modifying the potential energy and the dissipation function [29]. This scheme allows the total energy of the system to be shaped by using a feedback control law obtained from a Hamiltonian storage function [30].

\subsection{Hamiltonian Open Loop Dynamics of the System}

According to the PCB scheme, a matrix Hamiltonian notation of the primary system is required as

$$
\begin{aligned}
\dot{s} & =[J(s)-R(s)] \frac{\partial H(s)}{\partial s}+\beta(s) u \\
y & =\beta^{T}(s) \frac{\partial H(s)}{\partial s}
\end{aligned}
$$

where $s \in \Re^{n}$ includes all variables of the system energy, $J(s)=-J^{T}(s) \in \Re^{n x n}$ is an antisymmetric matrix that represents the conservative characteristic of the system, $R(s)=R^{T}(s) \in \Re^{n x n}$ is an asymmetric and positive semi-defined matrix which represents the dissipative characteristic, $H(s): \Re^{n} \rightarrow \Re$ is the storage energy function, $\beta \in \Re^{n}$ is a vector which defines the control input channels, $u \in \Re$ is the control input, and $y \in \Re$ is the system output. The energy balance for (33) is developed in [30] by

$$
\underbrace{H[s(\tau)]-H[s(0)]}_{\text {stored-energy }}+\underbrace{\left[\frac{\partial H(s)}{\partial s}\right]^{T} R(s)\left[\frac{\partial H(s)}{\partial s}\right]}_{\text {dissipated-energy }}=\underbrace{\int_{0}^{\tau} y^{T} u d s}_{\text {supplied-energy }}
$$

From (34) it can be observed that the sum between stored energy and dissipated energy results in the supplied energy, then it demonstrates that the primary system in (33) is dissipative passive. 
The system model in (7) is rewritten by using a change of variable such as $\vec{q}=\vec{z} \in \Re^{2}$ and $\vec{p}=M \dot{\vec{q}} \in \Re^{2}$, then, computing a Hamiltonian storage energy function defined by

$$
\begin{aligned}
H(\vec{q}, \vec{p})= & =\frac{1}{2} \vec{p}^{T} M^{-1} \vec{p}+\frac{1}{2} \vec{q}^{T} K \vec{q} \\
& =\frac{1}{2}\left[\begin{array}{ll}
\vec{q}^{T} & \vec{p}^{T}
\end{array}\right]\left[\begin{array}{cc}
K & 0 \\
0 & M^{-1}
\end{array}\right]\left[\begin{array}{l}
\vec{q} \\
\vec{p}
\end{array}\right]
\end{aligned}
$$

yields a Hamiltonian dynamics of the undisturbed system, as follows

$$
\left[\begin{array}{c}
\dot{\vec{q}} \\
\dot{\vec{p}}
\end{array}\right]=\left(\left[\begin{array}{cc}
0 & I \\
-I & 0
\end{array}\right]-\left[\begin{array}{cc}
0 & 0 \\
0 & C
\end{array}\right]\right) \underbrace{\left[\begin{array}{c}
K \vec{q} \\
M^{-1} \vec{p}
\end{array}\right]}_{=: \frac{\partial H(\vec{q}, \vec{p})}{\partial(\vec{q} \vec{p})}}+\left[\begin{array}{c}
0 \\
\vec{B}_{f}
\end{array}\right] u
$$

where $\vec{q}$ represents a vector of the generalized coordinates whereas $\vec{p}$ represents a vector of the generalized momenta of the system; $M, C, K, I \in \Re^{2 x 2}$ correspond to mass, damping, stiffness and identity matrices respectively; $\vec{B}_{f} \in \Re^{2}$ and $u \in \Re$.

The notation in (36) is in accordance with the general form of dissipative passive systems expressed by

$$
\dot{\vec{s}}=(J-R) \frac{\partial H(\vec{s})}{\partial \vec{s}}+\beta u
$$

where

$$
\frac{\partial H(\vec{s})}{\partial \vec{s}}=A \vec{s}, \vec{s}=\left[\begin{array}{ll}
\vec{q} & \vec{p}
\end{array}\right]^{T}, A=\left[\begin{array}{cc}
K & 0 \\
0 & M^{-1}
\end{array}\right], J=\left[\begin{array}{cc}
0 & I \\
-I & 0
\end{array}\right]=-J^{T}, R=\left[\begin{array}{ll}
0 & 0 \\
0 & C
\end{array}\right]=R^{T}, \beta=\left[\begin{array}{c}
0 \\
B_{f}
\end{array}\right]
$$

\subsection{Exact Regulation Error Dynamics Passive Output Feedback Control Design}

It is desired to minimize the dynamics of the generalized coordinates and momenta, $\min (\vec{q}, \vec{p})$, therefore $\min (\vec{s})$. According to the dissipative passive form in (37), a control law $u_{*}$ is proposed which is the reference input corresponding to the desired reference dynamics $\vec{s}_{*}$ and satisfies the system

$$
\dot{\vec{s}}_{*}=(J-R) \frac{\partial H\left(\vec{s}_{*}\right)}{\partial \vec{s}_{*}}+\beta u_{*}
$$

The regulation error and the control input error can be defined respectively as

$$
\vec{e}=\vec{s}-\vec{s}_{*} ; \quad e_{u}=u-u_{*}
$$

and the dynamics of exact regulation error is derived from (37) and (39) as follows

$$
\dot{\vec{e}}=(J-R) \frac{\partial H(\vec{e})}{\partial \vec{e}}+\beta e_{u}
$$

where

$$
\begin{aligned}
\frac{\partial H(\vec{e})}{\partial \vec{e}} & =A \vec{e}=A\left(\vec{s}-\vec{s}_{*}\right) \\
& =\frac{\partial H(\vec{s})}{\partial \vec{s}}-\frac{\partial H\left(\vec{s}_{*}\right)}{\partial \vec{s}_{*}} .
\end{aligned}
$$

Here, the Lyapunov stability criterion is considered to obtain a feedback control input law $u$, which is defined in terms of the control input error $e_{u}$, that allows asymptotic stability of the origin of the regulation error $\vec{e}$. A Hamiltonian candidate energy function of the regulation error dynamics is proposed as

$$
V(\vec{e})=H(\vec{e})=\frac{1}{2} \vec{e}^{T} A \vec{e}>0, \quad-\{0\}
$$


From a time derivative process of the proposed Lyapunov function $V(\vec{e})$, the inequality in (44) must be obtained to achieve asymptotic stability, which corresponds to a negative semi-defined function.

$$
\dot{V}(\vec{e})=\dot{H}(\vec{e}):=\vec{e}^{T} A \dot{\vec{e}}=\vec{e}^{T} A^{T} \dot{\vec{e}}=[A \vec{e}]^{T} \dot{\vec{e}} \Longrightarrow \dot{V}(\vec{e})=\left[\frac{\partial H(\vec{e})}{\partial \vec{e}}\right]^{T} \dot{\vec{e}} \leq 0
$$

Substituting the dynamics of the exact regulation error (41) in (44) yields

$$
\dot{V}(\vec{e})=\left[\frac{\partial H(\vec{e})}{\partial \vec{e}}\right]^{T}\left[(J-R) \frac{\partial H(\vec{e})}{\partial \vec{e}}+\beta e_{u}\right]
$$

and since $J$ is an antisymmetric matrix, then

$$
\left[\frac{\partial H(\vec{e})}{\partial \vec{e}}\right]^{T} J\left[\frac{\partial H(\vec{e})}{\partial \vec{e}}\right]:=0
$$

Finally, the error dynamics is computed as

$$
\dot{V}(\vec{e})=\underbrace{-\left[\frac{\partial H(\vec{e})}{\partial \vec{e}}\right]^{T} R\left[\frac{\partial H(\vec{e})}{\partial \vec{e}}\right]}_{\leq 0}+\left[\frac{\partial H(\vec{e})}{\partial \vec{e}}\right]^{T} \beta e_{u} .
$$

According with the definitions in (38), $R$ is a symmetric and positive semi-defined matrix, then the first term of the expression (47) is a negative semi-defined result. Consequently, to comply with the Lyapunov function inequality in (44), a dynamic $e_{u}$ in the second term is imposed with the form

$$
e_{u}=-\gamma \beta^{T}\left[\frac{\partial H(\vec{e})}{\partial \vec{e}}\right], \quad \gamma>0
$$

As such, according to Lyapunov theory, the regulation error converges asymptotically to the origin, bringing all states close to the desired reference. This control technique based on the passivity property is known as the exact regulation error dynamics passive output feedback (EREDPOF) controller design including suitable adaptive feed-forward precompensation [31]. Therefore, substituting (48) in (47), we have the following

$$
\begin{aligned}
\dot{V}(\vec{e}) & =-\left[\frac{\partial H(\vec{e})}{\partial \vec{e}}\right]^{T} R\left[\frac{\partial H(\vec{e})}{\partial \vec{e}}\right]-\left[\frac{\partial H(\vec{e})}{\partial \vec{e}}\right]^{T} \underbrace{\beta \gamma \beta^{T}}_{:=R_{d}}\left[\frac{\partial H(\vec{e})}{\partial \vec{e}}\right] \\
& =-\underbrace{\left[\frac{\partial H(\vec{e})}{\partial \vec{e}}\right]^{T}}_{:=\tilde{e}^{T}} \underbrace{\left(R+R_{d}\right)}_{:=\tilde{R}} \underbrace{\left[\frac{\partial H(\vec{e})}{\partial \vec{e}}\right]}_{:=\tilde{e}} \\
& =-\tilde{e}^{T} \tilde{R} \tilde{e} \leq 0
\end{aligned}
$$

where

$$
\tilde{R}=\left(\begin{array}{cc}
0 & 0 \\
0 & C+\gamma\left(B_{f}\right)^{2}
\end{array}\right)
$$

Using Sylvester's criterion, we verify $\tilde{R}$ is a positive semi-definite matrix. Thus (49) is a negative semi-definite function, and therefore the point of equilibrium of (39) under the control law (50) is stable. We take advantage of the asymptotic stability for autonomous systems (41), applying LaSalle's invariance principle, which for reasons of clarity, we give the following Theorem [32]. 
Theorem 1. Let $V(e): R^{n} \rightarrow R$ be such that on $\Omega_{c}=\left\{e \in R^{n}: V(e) \leq c\right\}$, a compact set we have $\dot{V}(e) \leq 0$. We define $S \subset \Omega_{c}$ by

$$
S=\left\{e \in R^{n}: \dot{V}(e) \leq 0\right\}
$$

Then, if $S$ contains no trajectories other than $x=0$ then 0 is asymptotically stable.

Since $\dot{V}(e)$, then $V(e)$ is a function decreasing of $t . V(e)$ is continuos in the compact set $\Omega_{c}$, and it is bounded inferiorly by $\Omega_{c}$. For example, satisfies $0 \leq V(e(0))$. Thus, $V(e)$ has a limit as $t \rightarrow \infty$. Hence $\dot{V}(e)=0$. Since $\Omega_{c}$ is an invariant set, $\dot{V}(e)=0$ and the only invariant solution is $e=0$. As the trivial solution is the unique solution of (41) restricted by $\Omega_{\mathcal{C}}$, then using Theorem 1, we can conclude that the origin of the state space is asymptotically stable.

Taking into account that $e_{u}=u-u_{*}$, so in terms of the generalized coordinates $\vec{q}$, the EREDPOF control law given by (48) guarantee the regulation of the system position and velocity variables. Thus, we have the following

$$
u=u_{*}-\left(\gamma B_{f}^{T}\right) \dot{\vec{e}}_{q}
$$

where $u_{*}$ is a feed-forward term, which is calculated by (39). Note also in (51), that $\gamma$ should be chosen in such a way that $u$ belong to $\left[f_{a}\left(i_{\min }\right), f_{a}\left(i_{\max }\right)\right]$ (see Equation (91)). For this, the $\gamma$ parameter was selected based on the following inequality

$$
0<\gamma<1
$$

To avoid a possible saturation in the actuator, we choose the $\gamma$ parameter smaller than 1 because this depends on the system's internal parameters.

The diagram of the proposed PBC scheme is shown in Figure 4.

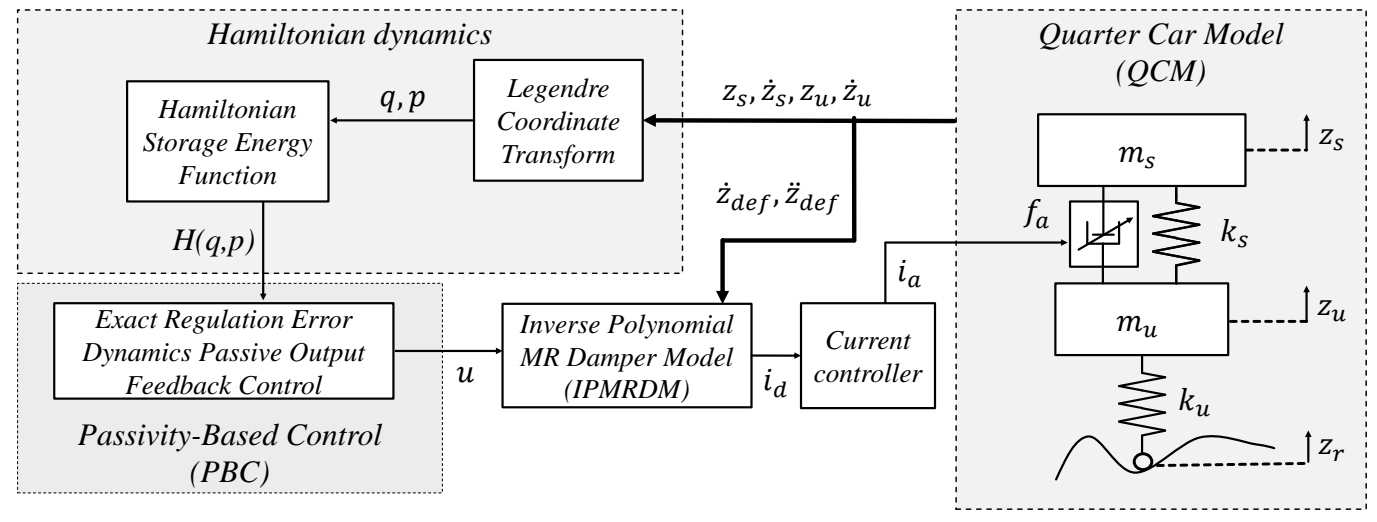

Figure 4. Proposed passivity-based control system for semi-active suspension with MRD.

\section{Disturbance Rejection Controller Design}

This control scheme allows for the indirect regulation of all system states by the regulation of the so-called flat output [33], thereby fulfilling both objectives of achieving comfort and road-holding. The controller robustness is improved by designing an extended state observer to estimate the unknown disturbances in order to be cancelled in the closed loop system.

By defining the state variables for the system (1) as $x_{1}=z_{s}, x_{2}=\dot{z}_{s}, x_{3}=z_{u}, x_{4}=\dot{z}_{u}$ and the control input as $u=f_{a}$ the system state space representation can be obtained as

$$
\begin{aligned}
\dot{x} & =A x+B u+E z_{r} \\
y & =C x
\end{aligned}
$$


where

$$
\begin{aligned}
x & =\left[\begin{array}{llll}
x_{1} & x_{2} & x_{3} & x_{4}
\end{array}\right]^{T} \\
A & =\left[\begin{array}{cccc}
0 & 1 & 0 & 0 \\
-\frac{k_{s}}{m_{s}} & -\frac{c_{s}}{m_{s}} & \frac{k_{s}}{m_{s}} & \frac{c_{s}}{m_{s}} \\
0 & 0 & 0 & 1 \\
\frac{k_{s}}{m_{u}} & \frac{c_{s}}{m_{u}} & \frac{-k_{s}-k_{u}}{m_{u}} & -\frac{c_{s}}{m_{u}}
\end{array}\right] \\
B & =\left[\begin{array}{llll}
0 & -\frac{1}{m_{s}} & 0 & \frac{1}{m_{u}}
\end{array}\right]^{T} \\
E & =\left[\begin{array}{llll}
0 & 0 & 0 & \frac{k_{u}}{m_{u}}
\end{array}\right]^{T}
\end{aligned}
$$

with $C \in \Re^{1 x 4}, u, y, z_{r} \in \Re$. The state vector (55) is assumed as completely measurable.

\subsection{Differential Flatness of the VMQC}

The system (53)-(54) is differentially flat, i.e., there is a system output $F(x)=y$ which accurately linearises the closed-loop system, which is the flat output. For a single inputsingle output (SISO) system, the flat output is defined as (59) by considering the full range controllability matrix $C_{M}(60)$ [33].

$$
\begin{aligned}
F(x) & =\left[\begin{array}{llll}
0 & 0 & 0 & 1
\end{array}\right] C_{M}^{-1} x \\
C_{M} & =\left[\begin{array}{llll}
B & A B & A^{2} B & A^{3} B
\end{array}\right]
\end{aligned}
$$

The differential flatness property allows for the system states $x_{i}, i=1, \ldots, 4$, as well as the control law $u$ to be parametrized in terms of the flat output $F$ and a finite number of its successive time derivatives, as follows

$$
\begin{aligned}
\dot{F} & =f_{1}\left(x_{1}, x_{2}, x_{3}, x_{4}\right) \\
\ddot{F} & =f_{2}\left(x_{1}, x_{2}, x_{3}, x_{4}\right) \\
\dddot{F} & =f_{3}\left(x_{1}, x_{2}, x_{3}, x_{4}\right) \\
F^{(4)} & =\xi\left(x_{1}, x_{2}, x_{3}, x_{4}, t\right)+u
\end{aligned}
$$

where $\xi$ represents a function including the endogenous and exogenous unknown timevariant system disturbances. The existence of a real constant $\xi_{0}$ is considered, such as the function $\xi$ which is bounded as $|\xi| \leq \xi_{0}$.

\subsection{Disturbance Rejection Control}

A disturbance rejection control (DRC) is designed through the differential parametrization of the system (64), in combination with a linear extended state observer, which will be responsible for estimating the endogenous and exogenous perturbations of the system. Thus, the DRC is designed as

$$
\begin{aligned}
& u=v(\dddot{e}, \ddot{e}, \dot{e}, e)-\xi \\
& v=-k_{3} \dddot{e}-k_{2} \ddot{e}-k_{1} \dot{e}-k_{0} e
\end{aligned}
$$

where, the regulation errors are defined as

$$
e=F-\bar{F}, \quad \dot{e}=\dot{F}-\dot{\bar{F}}, \quad \ddot{e}=\ddot{F}-\ddot{\bar{F}}, \quad \dddot{e}=\dddot{F}-\ddot{\bar{F}}
$$

while $\xi$ is the unknown perturbation function; $v$ is the virtual control, which imposes the regulation error dynamics in closed loop, its desired references are given as: $\ddot{\bar{F}}=\ddot{\bar{F}}=$ $\dot{\bar{F}}=\bar{F}=0$. The set of coefficients $\left\{k_{3}, k_{2}, k_{1}, k_{0}\right\}$ are positive values which are chosen with the help of a desired closed loop fourth-degree characteristic polynomial of $p_{d}(s)=$ 
$\left(s^{2}+2 \zeta_{c} \omega_{c} s+\omega_{c}^{2}\right)^{2}$. Consequently, $\lambda_{3}=4 \zeta_{c} \omega_{c}, \lambda_{2}=\left(2 \omega_{c}^{2}+4 \zeta_{c}^{2} \omega_{c}^{2}\right), \lambda_{1}=4 \zeta_{c} \omega_{c}^{3}$, and $\lambda_{0}=\omega_{c}^{4}$. Therefore, the regulation error stability in closed loop has an asymptotic convergence near to zero.

\subsection{Linear Extended State Observer (LESO) Design for the DRC}

From (64), the following linear extended state observer (LESO) is designed as follows

$$
\begin{aligned}
& \dot{y}_{1}=y_{2}+\lambda_{5}\left(y-y_{1}\right) \\
& \dot{y}_{2}=y_{3}+\lambda_{4}\left(y-y_{1}\right) \\
& \dot{y}_{3}=y_{4}+\lambda_{3}\left(y-y_{1}\right) \\
& \dot{y}_{4}=\gamma_{1}+u+\lambda_{2}\left(y-y_{1}\right) \\
& \dot{\gamma}_{1}=\gamma_{2}+\lambda_{1}\left(y-y_{1}\right) \\
& \dot{\gamma}_{2}=\lambda_{0}\left(y-y_{1}\right)
\end{aligned}
$$

where, $y=F$ is the flat output of the system, which is calculated by means of (59); $y_{1}=\hat{F}$ is the estimated value of the flat output, while $y_{2}=\dot{\hat{F}}, y_{3}=\ddot{\hat{F}}$ and $y_{4}=\dddot{\hat{F}}$ are the estimated values of the successive time derivatives of the flat output; $\gamma_{1}=\hat{\xi}$ is the estimation of the unknown perturbation function, and $\gamma_{2}=\dot{\hat{\xi}}$ is the estimated time derivative of the perturbation function, which are considered as unknown.

To demonstrate the closed-loop stability of the system, we will analyze the stability of the linear extended state observer and the disturbance rejection controller.

\subsection{Closed-Loop Stability Analysis}

We present here the proof of stability of the LESO's output estimation error dynamics $[34,35]$. Defining the output estimation error as

$$
e_{o b s}=y-y_{1}
$$

whose first time-derivative is given by

$$
\dot{e}_{o b s}=\dot{y}-\dot{y}_{1}=\left(\dot{F}-y_{2}\right)-\lambda_{5} e_{o b s}
$$

Finding the time-derivative of (75)

$$
\ddot{e}_{o b s}+\lambda_{5} \dot{e}_{o b s}=\ddot{F}-y_{3}-\lambda_{4} e_{o b s}
$$

The third time-derivative of $e_{o b s}$, is obtained by

$$
\dddot{e}_{o b s}+\lambda_{5} \ddot{e}_{o b s}+\lambda_{4} \dot{e}_{o b s}+\lambda_{3} e_{o b s}=\dddot{F}-y_{4}
$$

The fourth time-derivative of $e_{o b s}$ can be expressed by

$$
\stackrel{(4)}{e}_{o b s}+\lambda_{5} \dddot{e}_{o b s}+\lambda_{4} \ddot{e}_{o b s}+\lambda_{3} \dot{e}_{o b s}+\lambda_{2} e_{o b s}=\xi\left(x_{1}, x_{2}, x_{3}, x_{4}, t\right)-\gamma_{1}
$$

The fifth time-derivative of $e_{o b s}$ is

$$
\stackrel{(5)}{e}_{o b s}+\lambda_{5} e_{o b s}^{(4)}+\lambda_{4} \dddot{e}_{o b s}+\lambda_{3} \ddot{e}_{o b s}+\lambda_{2} \dot{e}_{o b s}+\lambda_{1} e_{o b s}=\dot{\xi}\left(x_{1}, x_{2}, x_{3}, x_{4}, t\right)-\gamma_{2}
$$

Finally, the estimation error dynamics is given by

$$
\stackrel{(6)}{e}_{o b s}+\lambda_{5} e_{o b s}^{(5)}+\lambda_{4} e_{o b s}^{(4)}+\lambda_{3} \dddot{e}_{o b s}+\lambda_{2} \ddot{e}_{o b s}+\lambda_{1} \dot{e}_{o b s}+\lambda_{0} e_{o b s}=\ddot{\xi}\left(x_{1}, x_{2}, x_{3}, x_{4}, t\right)
$$

Since $\ddot{\xi}\left(x_{1}, x_{2}, x_{3}, x_{4}, t\right)$ is assumed to be uniformly absolutely bounded, then there exist design coefficients $\left\{\lambda_{5}, \lambda_{4}, \lambda_{3}, \lambda_{2}, \lambda_{1}, \lambda_{0}\right\}$ for the LESO's (in the form of GPI observers), 
such that the estimation errors are uniformly asymptotically convergent to a small neighborhood of the origin of the phase space of the estimation error dynamics. The faster the estimation error dynamics, the smaller the convergence neighborhood of the origin. The value of the gain parameters is customarily chosen via a term-by-term comparison of (80) with those of a desired, nominal, dominating, sixth-degree characteristic polynomial $p_{d-o b s}(s)=\left(s^{2}+2 \zeta_{o} \omega_{0} s+\omega_{o}^{2}\right)^{3}$. Therefore, $\lambda_{5}=6 \zeta_{0} \omega_{0}, \lambda_{4}=\left(3 \omega_{0}^{2}+12 \zeta_{0}^{2} \omega_{o}^{2}\right)$, $\lambda_{3}=\left(8 \zeta_{0}^{3} \omega_{0}^{3}+12 \zeta_{0} \omega_{0}^{3}\right), \lambda_{2}=\left(3 \omega_{0}^{4}+12 \zeta_{0}^{2} \omega_{0}^{4}\right), \lambda_{1}=6 \zeta_{0} \omega_{0}^{5}$, and $\lambda_{0}=\omega_{0}^{6}$.

On the other hand, from (64)-(66) We have the dynamics system of the following form

$$
F^{(4)}=\xi\left(x_{1}, x_{2}, x_{3}, x_{4}, t\right)+u
$$

with the disturbance rejection controller as

$$
u=-k_{3} \dddot{\hat{F}}-k_{2} \ddot{\hat{F}}-k_{1} \dot{\hat{F}}-k_{0}(\hat{F}-\bar{F})-\gamma_{1}
$$

Substituting (82) in (81), we have the closed-loop system of the following form

$$
F^{(4)}=\underbrace{\xi\left(x_{1}, x_{2}, x_{3}, x_{4}, t\right)-\gamma_{1}}_{:=\Delta_{p}\left(e, e_{o b s}\right)}-k_{3} \dddot{\hat{F}}-k_{2} \ddot{\hat{F}}-k_{1} \dot{\hat{F}}-k_{0}(\hat{F}-\bar{F})
$$

The estimated values of $\hat{F}, \dot{\hat{F}}, \ddot{\hat{F}}$, and $\ddot{\hat{F}}$ are defined in terms of the observation error, the flat output, and successive time-derivatives

$$
\hat{F}=F-e_{o b s}, \dot{\hat{F}}=\dot{F}-\dot{e}_{o b s}, \ddot{\hat{F}}=\ddot{F}-\ddot{e}_{o b s}, \ddot{\hat{F}}=\dddot{F}-\dddot{e}_{o b s}
$$

The regulation error dynamics given by (83) is transformed as follow

$$
\stackrel{(4)}{e}=-k_{3}\left(\dddot{e}-\dddot{e}_{o b s}\right)-k_{2}\left(\ddot{e}-\ddot{e}_{o b s}\right)-k_{1}\left(\dot{e}-\dot{e}_{o b s}\right)-k_{0}\left(e-e_{o b s}\right)+\Delta_{p}\left(e, e_{o b s}\right)
$$

Therefore, the closed-loop system is expressed in terms of the tracking error, observation error, and perturbation error

$$
\stackrel{(4)}{e}+k_{3} \dddot{e}+k_{2} \ddot{e}+k_{1} \dot{e}+k_{0} e=k_{3} \dddot{e}_{o b s}+k_{2} \ddot{e}_{o b s}+\ddot{k}_{1} \dot{e}_{o b s}+k_{0} e_{o b s}+\Delta_{p}\left(e, e_{o b s}\right)
$$

As the regulation error and observation error are linear, then we can apply the Laplace transformation with initial conditions equal to zero, thus we have

$$
\left(s^{4}+k_{3} s^{3}+k_{2} s^{2}+k_{1} s+k_{0}\right) e(s)=\left(s^{4}+k_{3} s^{3}+k_{2} s^{2}+k_{1} s+k_{0}\right) e_{o b s}(s)+\Delta_{p}(s)
$$

The closed-loop system can be expressed as a transfer function with two inputs

$$
e(s)=\frac{\left(s^{4}+k_{3} s^{3}+k_{2} s^{2}+k_{1} s+k_{0}\right)}{\left(s^{4}+k_{3} s^{3}+k_{2} s^{2}+k_{1} s+k_{0}\right)} e_{o b s}(s)+\frac{1}{\left(s^{4}+k_{3} s^{3}+k_{2} s^{2}+k_{1} s+k_{0}\right)} \Delta_{p}(s)
$$

To analyze the stability in the closed-loop of the system, we appeal to the steady-state error, let by the final value theorem

$$
e_{s s}=\lim _{t \rightarrow \infty} e(t)=\lim _{s \rightarrow 0} s e(s)=\lim _{s \rightarrow 0} \frac{s\left(s^{4}+k_{3} s^{3}+k_{2} s^{2}+k_{1} s+k_{0}\right) e_{o b s}(s)}{\left(s^{4}+k_{3} s^{3}+k_{2} s^{2}+k_{1} s+k_{0}\right)}+\frac{s \Delta_{p}(s)}{\left(s^{4}+k_{3} s^{3}+k_{2} s^{2}+k_{1} s+k_{0}\right)}
$$

The summarizes of the steady-state error results for each disturbance input are the following: 


$$
e_{s s}:=\left\{\begin{array}{c}
0 \quad \text { if } \quad e_{o b s}(s)=\Delta_{p}(s)=1 \\
1 \quad \text { if } e_{o b s}(s)=\Delta_{p}(s)=1 / s \\
0 \quad \text { if } \quad e_{o b s}(s)=\Delta_{p}(s)=\frac{w}{s^{2}+w^{2}}
\end{array}\right.
$$

Remark 3. The regulation error is asymptotically stable near-zero whenever the controller's tuned is made utilizing a fourth-order Hurwitz polynomial.

Remark 4. The estimation or observation errors are uniformly asymptotically convergent to a small neighborhood of the phase space's origin of the estimation error dynamics. The faster the estimation error dynamics, the smaller the convergence neighborhood of the origin. This, whenever the LESO tune is made employing a Hurwitz polynomial of sixth order.

Remark 5. The tuned of the controller and LESO observer is made by separated. The controller's robustness resides in the estimation and reduction to the same time as the internal and external disturbances unknown by the system.

The proposed global system (DRC+LESO) is illustrated in Figure 5.

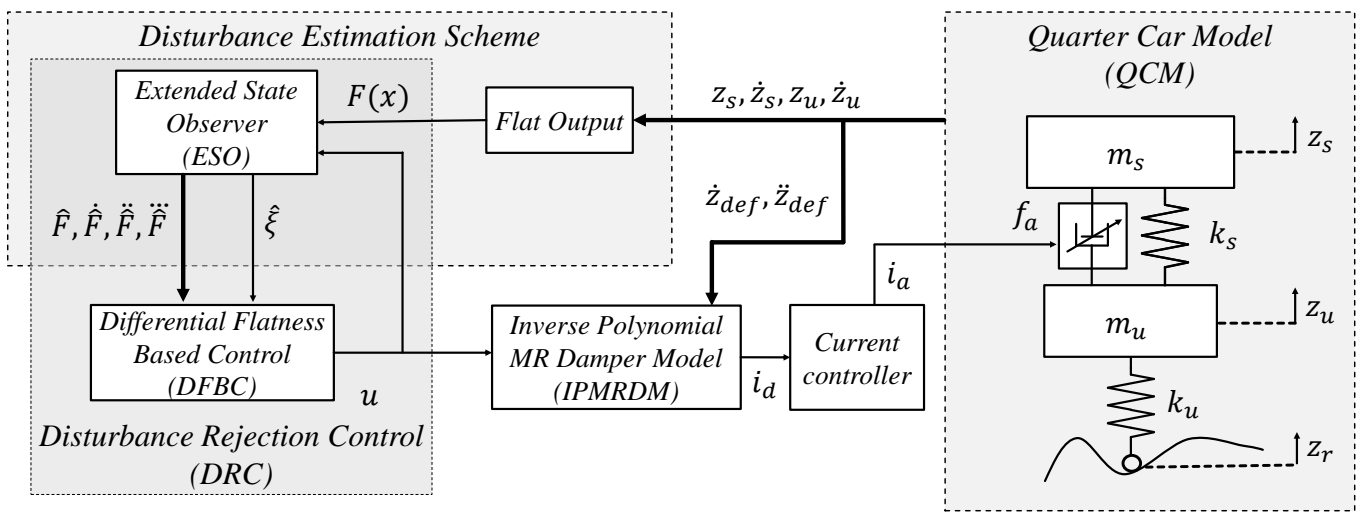

Figure 5. DRC system for semi-active suspension with MRD.

\section{Results}

The methodology of analysis for automotive suspensions reported by Savaresi et al. [7] is implemented in this section to evaluate the proposed SAC schemes. The comfort and road-holding performance of the suspension can be evaluated with the Transmissibility Frequency Response (TFR) of the sprung mass displacement $Z_{S}(f)$ and the tyre deflection displacement $Z_{\text {deft }}(f)$. The comfort and road-holding performance criteria are defined as [7]

$$
\underbrace{I_{\mathcal{C}}=\frac{J\left(Z_{s}^{\text {nom }}(f)\right)}{J\left(Z_{s}(f)\right)}}_{\text {comfort }}, \quad \underbrace{I_{r h}=\frac{J\left(Z_{\text {deft }}^{\text {nom }}(f)\right)}{J\left(Z_{\text {deft }}(f)\right)}}_{\text {road-holding }}
$$

where $Z_{s}^{\text {nom }}(f)$ and $Z_{\text {deft }}^{\text {nom }}(f)$ are the TFR for sprung mass and tyre deflection displacements respectively, both developed by a constant nominal current of MRD; $J(Y(f))$ is the root main square (RMS) function of TFR of interest $Y(f)$. The criteria in (89) describes the overall suspension performance as follows:

- $\quad$ if $I_{\mathcal{C}}>1$ (resp. $I_{\mathcal{C}}<1$ ) then the analysed suspension is more (resp. less) comfortable than the nominal reference one.

- $\quad$ if $I_{r h}>1$ (resp. $I_{r h}<1$ ) then the analysed suspension provides better (resp. worse) road-holding performance than the nominal reference one. 
The TFR is estimated frequency by frequency (Algorithm 1 in the methodology of Savaresi et al. [7]) using a sinusoidal perturbation function, which is applied to the system during $P$ periods of this signal as $z_{r}=Z_{r} \sin (2 \pi f t)$, where $f \in\left[f_{\min }, f_{\text {max }}\right], t \in$ $\left[P / f_{\min }, P / f_{\max }\right]$. For each response $y(t)$ the maximum transmissibility value is calculated by $Y(f)=20 \log \left\{\max [y(t)] / \max \left[z_{r}(t, f)\right]\right\}$. For more details of this methodology, refer to chapter 4 from [7]. In addition, a parameter disturbance is applied by means of two sprung mass variations carried out at $\frac{P}{3}$ and $\frac{2 P}{3}$ time as

$$
m_{s}=\left\{\begin{array}{cc}
m_{\min } & t<\frac{P}{3} \\
m_{\text {nom }} & \frac{2 P}{3} \geq t \geq \frac{P}{3} \\
m_{\max } & t>\frac{2 P}{3}
\end{array}\right\} .
$$

A MR suspension study case with numerical parameters in Table 2 is considered to verify comfort and road-holding performance under different damping control inputs, which uses the inverse polynomial MR damper model expressed in (6) to compute the electric current that provides the desired damping force. Furthermore, a current regulation is carried out by a PID controller to track the computed current. The numerical results are obtained through MATLAB/Simulink simulations using ODE4 method. The numerical values corresponding to masses, springs constant and damping constant are proposed based on the experimental test rig described in [36] which was designed taking into account the characteristics of the MRD, particulary the maximum damping force available. The disturbance frequency range is defined in order to excite the two modal frequencies of the suspension system.

Table 2. Parameters for numerical simulation of the closed-loop system with the proposed controllers.

\begin{tabular}{ccc}
\hline Parameter & Description & Value \\
\hline$m_{\min }$ & Minimum sprung mass & $100 \mathrm{~kg}$ \\
$m_{\text {nom }}$ & Nominal sprung mass & $135 \mathrm{~kg}$ \\
$m_{\max }$ & Maximum sprung mass & $187.5 \mathrm{~kg}$ \\
$m_{u}$ & Unsprung mass & $27.8 \mathrm{~kg}$ \\
$k_{s}$ & Suspension stiffness & $18.775 \mathrm{kN} / \mathrm{m}$ \\
$k_{u}$ & Tire stiffness & $148.2886 \mathrm{kN} / \mathrm{m}$ \\
$c_{s}$ & Suspension damping & $100 \mathrm{Ns} / \mathrm{m}$ \\
$i_{l i m}$ & Maximum input current permissible for MRD & $2 \mathrm{~A}$ \\
$f_{o}$ & Maximum damping force provided by MRD & $1750 \mathrm{~N}$ \\
$f_{\max }$ & Upper test frequency & $30 \mathrm{~Hz}$ \\
$f_{\min }$ & Lower test frequency & $1 \mathrm{~Hz}$ \\
$Z_{r}$ & Disturbance signal amplitude & $1 \mathrm{~cm}$ \\
$P$ & Test periods & 10 \\
$\omega_{f}$ & PPF Frequency & $12 \mathrm{rad} / \mathrm{s} \mathrm{or} \mathrm{72} \mathrm{rad/s}$ \\
$\zeta_{f}$ & PPF Damping ratio & 1.0 \\
$g$ & PPF gain constant & $5.0 \mathrm{or} 1.0$ \\
$\omega_{i}$ & $i$-th MPPF Frequency & $12 \mathrm{rad} / \mathrm{s} \mathrm{and} 72 \mathrm{rad} / \mathrm{s}$ \\
$\zeta_{i}$ & $i$-th MPPF Damping ratio & 1.0 \\
$g_{i}$ & $i$-th MPPF gain constant & $5.0 \mathrm{and} 1.0$ \\
$\gamma$ & PBC gain constant & 0.44 \\
$\omega_{c}$ & Angular frequency for DRC scheme & $20 \mathrm{rad} / \mathrm{s}$ \\
$\zeta_{c}$ & Damping ratio for DRC scheme & 3.0 \\
$\omega_{0}$ & Angular frequency for LESO & $150 \mathrm{rad} / \mathrm{s}$ \\
$\zeta_{o}$ & Damping ratio for LESO & 4.0 \\
$T$ & Sample time for simulation & $100 \mathrm{\mu s}$ \\
\hline
\end{tabular}

The system response under constant values of current input $\left\{i_{\max }, i_{\text {nom }}, i_{\min }\right\}$ is analyzed to compare the overall suspension performance with the proposed controllers. The TFR of the system under different inputs for MR current is illustrated by Figures 6 and 7, 
where two vibration modes can be located in $f_{1} \approx 2 \mathrm{~Hz}\left(\omega_{1} \approx 12 \mathrm{rad} / \mathrm{s}\right)$ and $f_{2} \approx 12 \mathrm{~Hz}$ $\left(\omega_{2} \approx 72 \mathrm{rad} / \mathrm{s}\right)$. It can be observed that: if the maximum (resp. minimum) electric current is applied, then $Z_{s}(f)$ and $Z_{\text {deft }}(f)$ TFR are attenuated (resp. intensified) around to $f_{1}$ and $f_{2}$, but both are intensified (resp. attenuated) in other frequencies.

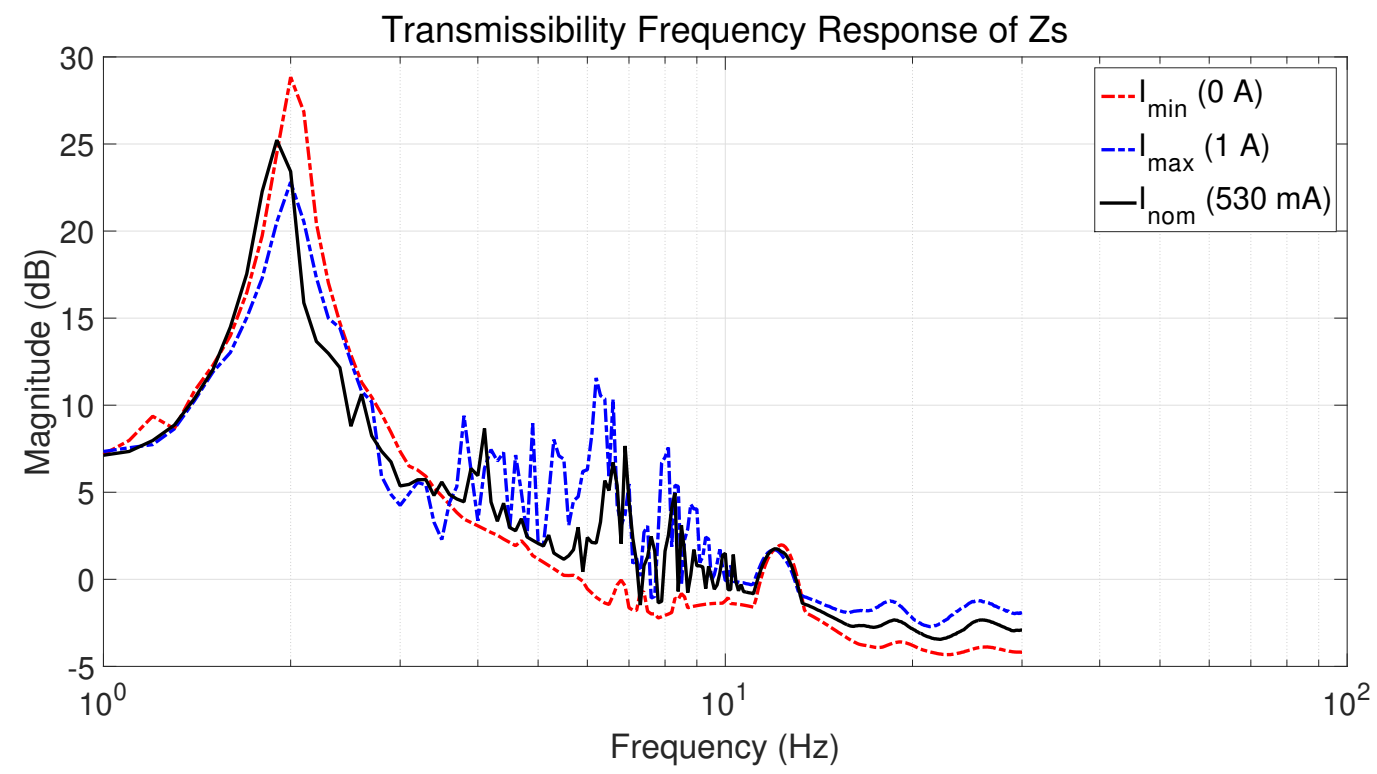

Figure 6. Comparison of Transmissibility Frequency Response (TFR) of the sprung mass displacement for different current inputs.

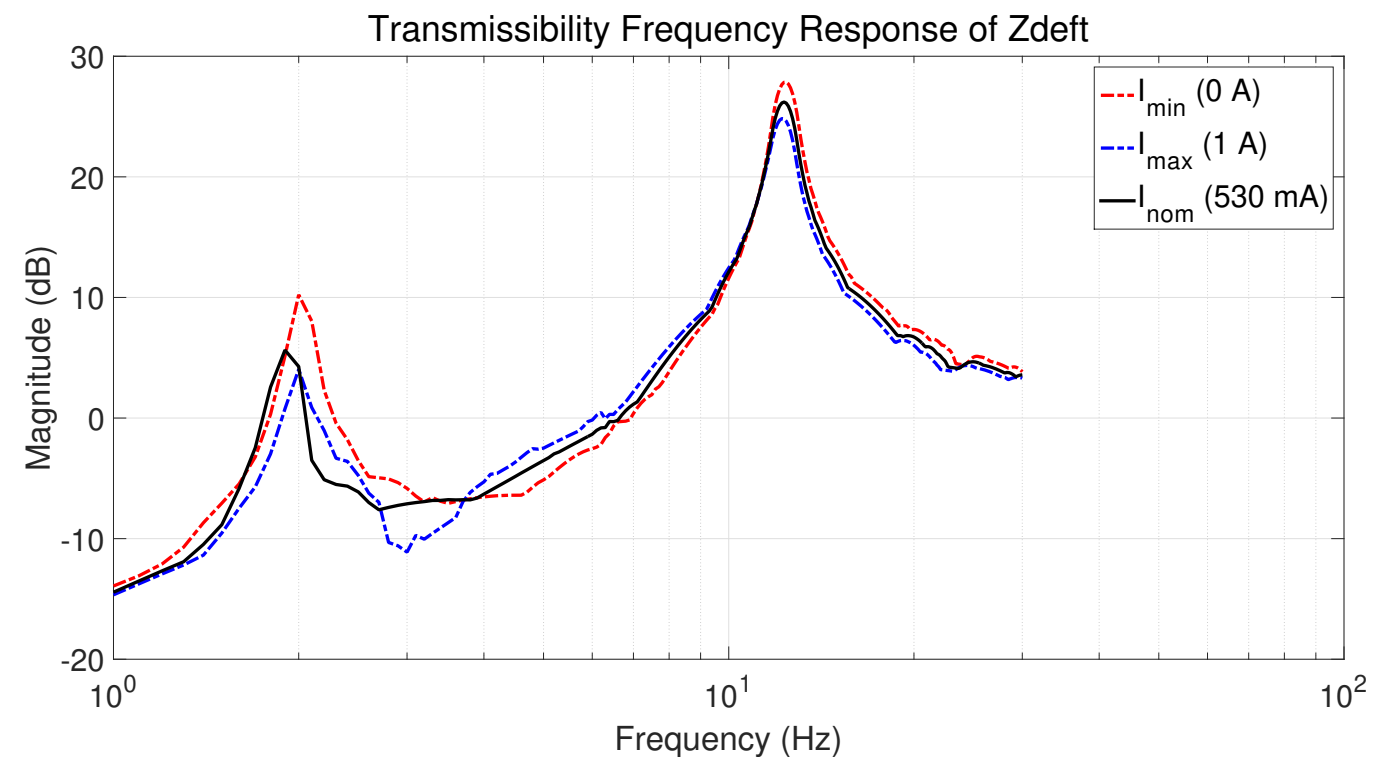

Figure 7. Comparison of TFR of the tyre deflection for different current inputs.

The effectiveness of three control schemes proposed in this work is compared through numerical simulation. Firstly, the PPF scheme expressed in Equations (10) and (11) is designed for two different objectives: first vibration mode improvement (low frequencies) and second vibration mode improvement (high frequencies). Moreover, the MPPF scheme in (20) is proposed to attenuate the motion amplitudes in both vibration modes simultaneously as described in Section 3. Secondly, a positive high gain for an exact regulation error dynamics passive output feedback controller is considered to quickly bring the system dynamics close to the stationary reference following the methodology of Section 4. Next, both differential flatness-based control and extended state observer (DRC scheme) are proposed 
as an effective alternative for unknown disturbances rejection increasing the robustness and reliability of the system. To observe the exogenous disturbances, the angular frequency for LESO is set faster than the angular for DRC scheme (see the development in Section 5 and parameters in Table 2). Additionally, a classic semi-active Skyhook (SH) control is implemented to compare the overall suspension performance of the proposed controllers in this work, as follows

$$
u_{S H}=\left\{\begin{array}{ll}
f_{a}\left(i_{\text {max }}\right) & \left(\dot{z}_{s}-\dot{z}_{u}\right) \dot{z}_{s} \geq 0 \\
f_{a}\left(i_{\text {min }}\right) & \left(\dot{z}_{s}-\dot{z}_{u}\right) \dot{z}_{s}<0
\end{array}\right\} .
$$

where $f_{a}\left(i_{\max }\right)$ and $f_{a}\left(i_{\min }\right)$ are the damping forces developed by the minimum and maximum current, respectively [7].

Figures 8 and 9 show the TFR of the sprung mass displacement and the tyre deflection for the closed-loop system with the controllers described above.

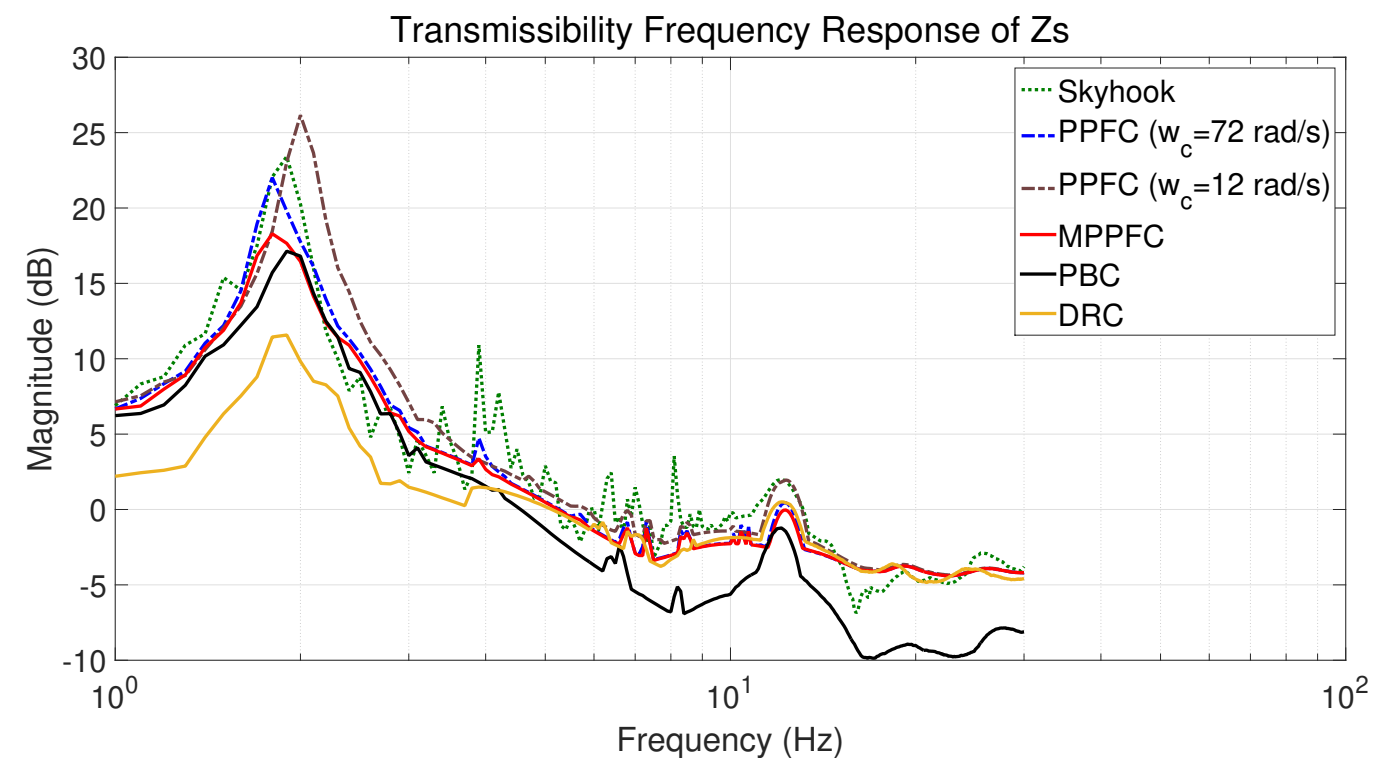

Figure 8. Comparison of TFR of the sprung mass displacement for different damping control.

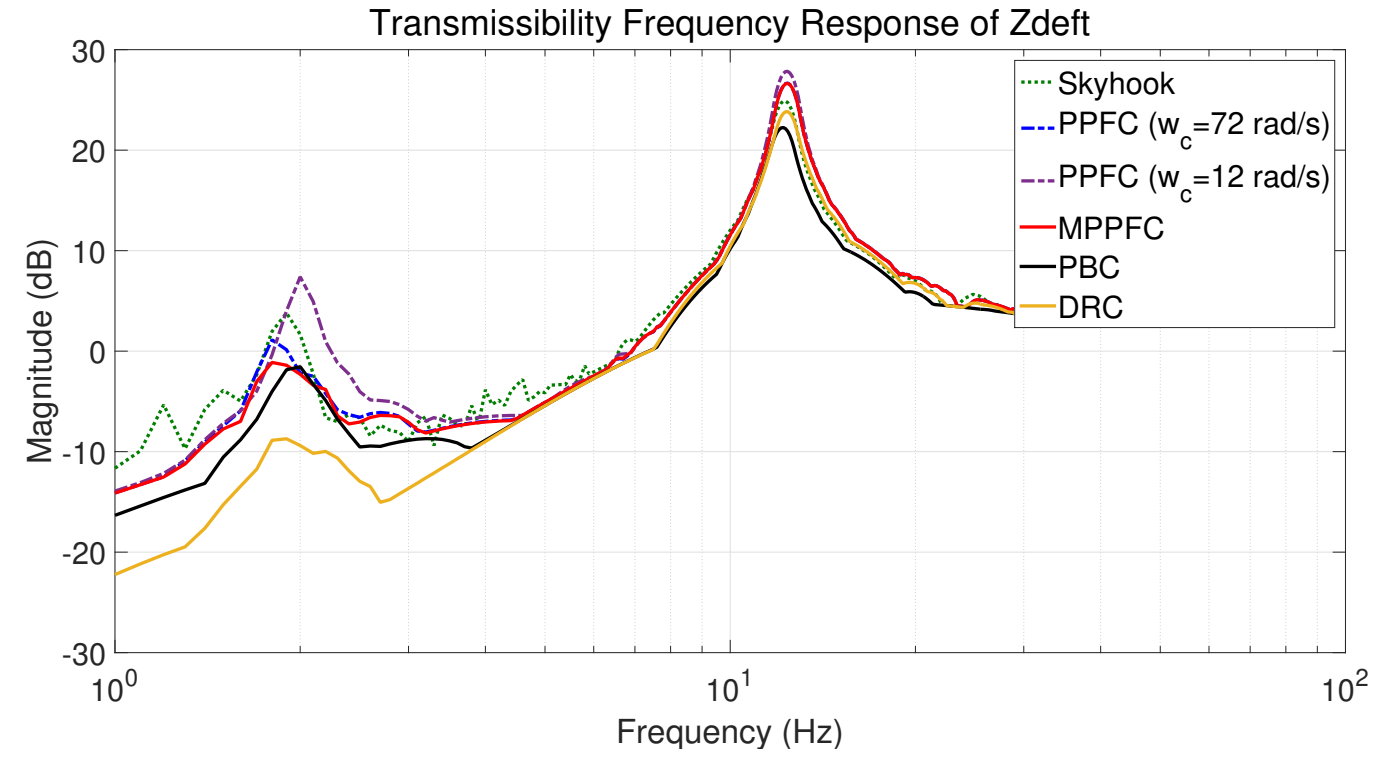

Figure 9. Comparison of TFR of the tyre deflection for different damping control.

To quantitatively compare the controllers' performance, the criteria given by Equation (89) were normalized regarding the suspension system with MRD operating with a 
constant nominal current $(530 \mathrm{~mA})$. The obtained results of normalized indexes evaluation for both comfort and road-holding performance are presented in Table 3.

Table 3. Normalized performance criteria comparison for different damping control.

\begin{tabular}{ccc}
\hline Controller & Comfort & Road-Holding \\
\hline$i_{\text {nom }}=530 \mathrm{~mA}$ & 1.0 & 1.0 \\
$i_{\min }=0 \mathrm{~A}$ & 0.5766 & 0.7324 \\
$i_{\text {max }}=1 \mathrm{~A}$ & 1.1262 & 1.2723 \\
Skyhook scheme & 1.3242 & 1.2379 \\
PPF (comfort approach) & 0.8866 & 0.7365 \\
PPF (road-holding approach) & 1.6428 & 0.8981 \\
MPPF scheme & 2.2926 & 0.9012 \\
Passivity-based scheme & 3.1954 & 1.9634 \\
DRC scheme & 5.1122 & 1.4534 \\
\hline
\end{tabular}

As one can observe, a soft suspension represented by open loop system operating at $i_{\text {min }}$ is the worst evaluated in both criteria. On the other hand a rigid suspension operating at $i_{\text {max }}$ improve both performance indexes. However, MRD manufacturer suggest not operate it by an extended period of time at maximum current. Besides, in Figures 6 and 7 a poor performance of the rigid suspension at intermediate frequency range can be appreciated. Regarding to closed-loop system with the classical sky-hook controller and the proposed SAC schemes, modal controls are unable to improve simultaneously comfort and road-holding indexes, and only the PCB and DRC schemes improve both criteria, with the first one producing the best performance at high frequencies (road-holding) operation, and the second one producing a superior performance at low frequencies (comfort) region.

Changes in sprung mass described in Equation (90) were introduced to verify the effect of these variation in the performance of the proposed controllers. As we mentioned above, parameters of the primary system are defined according to a experimental test ring. The range of variation for the sprung mass represents in a proportional manner, different number of passengers in the vehicle keeping in mind that the sprung mass value is selected according to the physical limitations on the MRD. Results for the TFR of the sprung mass displacement and the tyre deflection with this disturbance in the mass value are shown in Figures 10 and 11.

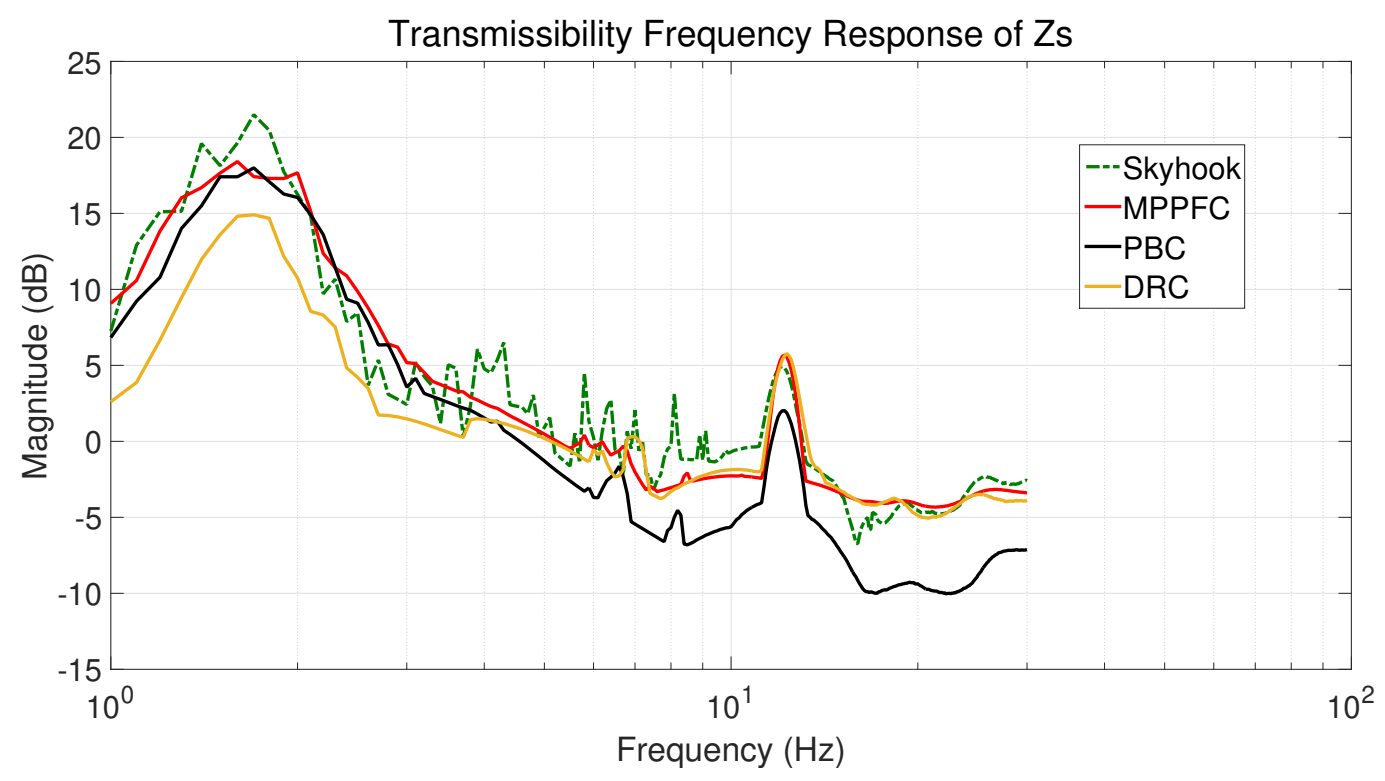

Figure 10. Comparison of TFR of the sprung mass displacement for different damping control and variable sprung mass. 


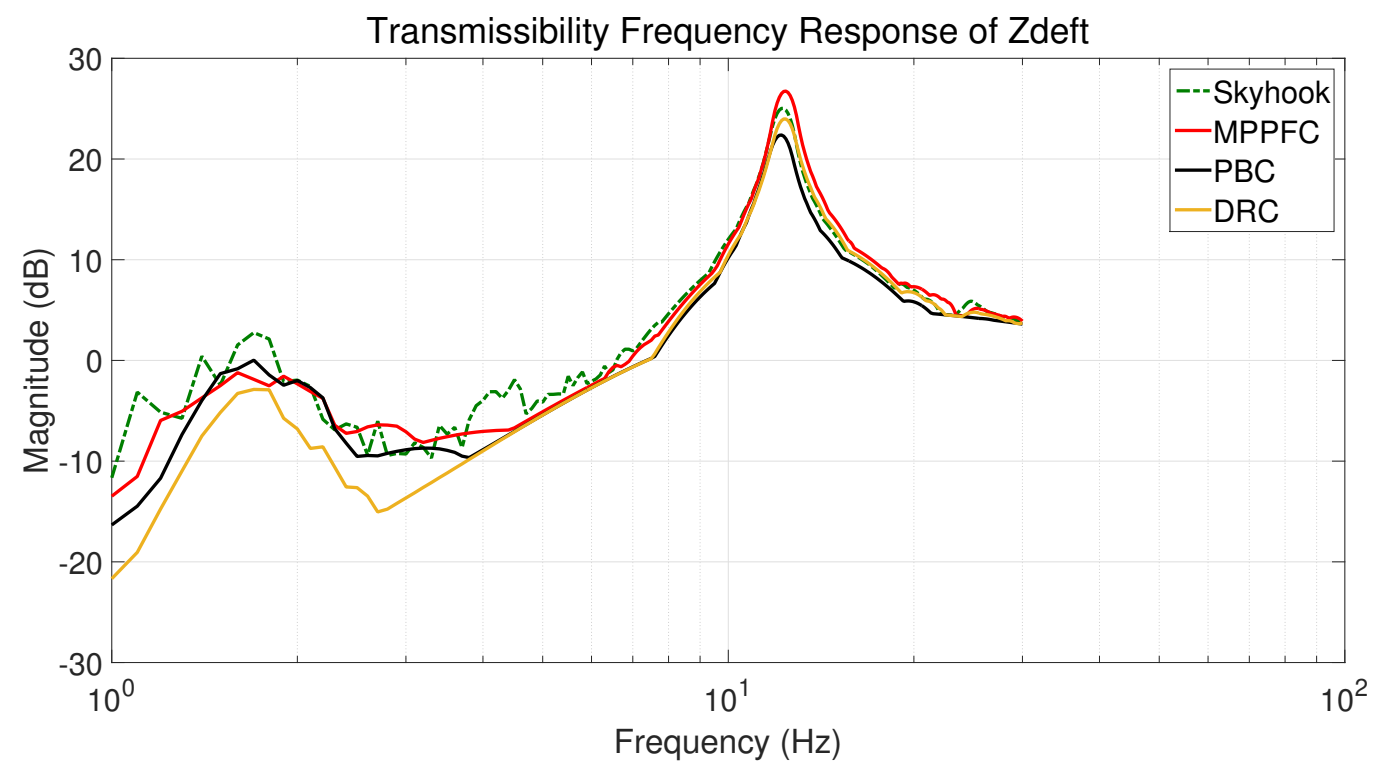

Figure 11. Comparison of TFR of the tyre deflection for different damping control and variable sprung mass.

Normalized criteria evaluation for variable sprung mass is shown in Table 4. As can be observed, road-holding index practically does not have any change in comparison with results in Table 4. Regarding comfort index, the rigid suspension is considerably affected by the sprung mass variation resulting in a worst performance in relation with the reference case (open-loop system with nominal current input). Again, DRC and PBC present the best performance at low and high frequencies respectively, even with disturbances in the sprung mass value. Moreover, these control schemes achieve the attenuation at the two main vibration modes.

Table 4. Normalized performance criteria comparison for different damping control and variable sprung mass.

\begin{tabular}{ccc}
\hline Controller & Comfort & Road-Holding \\
\hline$i_{\text {nom }}=530 \mathrm{~mA}$ & 1.0 & 1.0 \\
$i_{\min }=0 \mathrm{~A}$ & 0.5747 & 0.7352 \\
$i_{\max }=1 \mathrm{~A}$ & 0.8047 & 1.2691 \\
Skyhook scheme & 1.4319 & 1.2359 \\
PPF (comfort approach) & 0.8602 & 0.7396 \\
PPF (road-holding approach) & 1.1997 & 0.9011 \\
MPPF scheme & 1.8206 & 0.9050 \\
Passivity-based scheme & 2.3945 & 1.9672 \\
DRC scheme & 3.6119 & 1.4550 \\
\hline
\end{tabular}

Finally, Figures 12 and 13 present the maximum damping force and the power consumption of the MRD for each proposed SAC scheme corresponding to results in Figures 8 and 9 and Table 3. These values are calculated for each frequency of the proposed disturbance. The obtained damping forces are within the range of maximum force provided by the MRD considered in this work. Moreover, the MRD reaches saturation in frequencies around the second vibration mode, as can be observed in Figure 13. Despite this operation condition, the MRD is able to provide the damping force requested to attenuate the TFR in both vibration modes. 


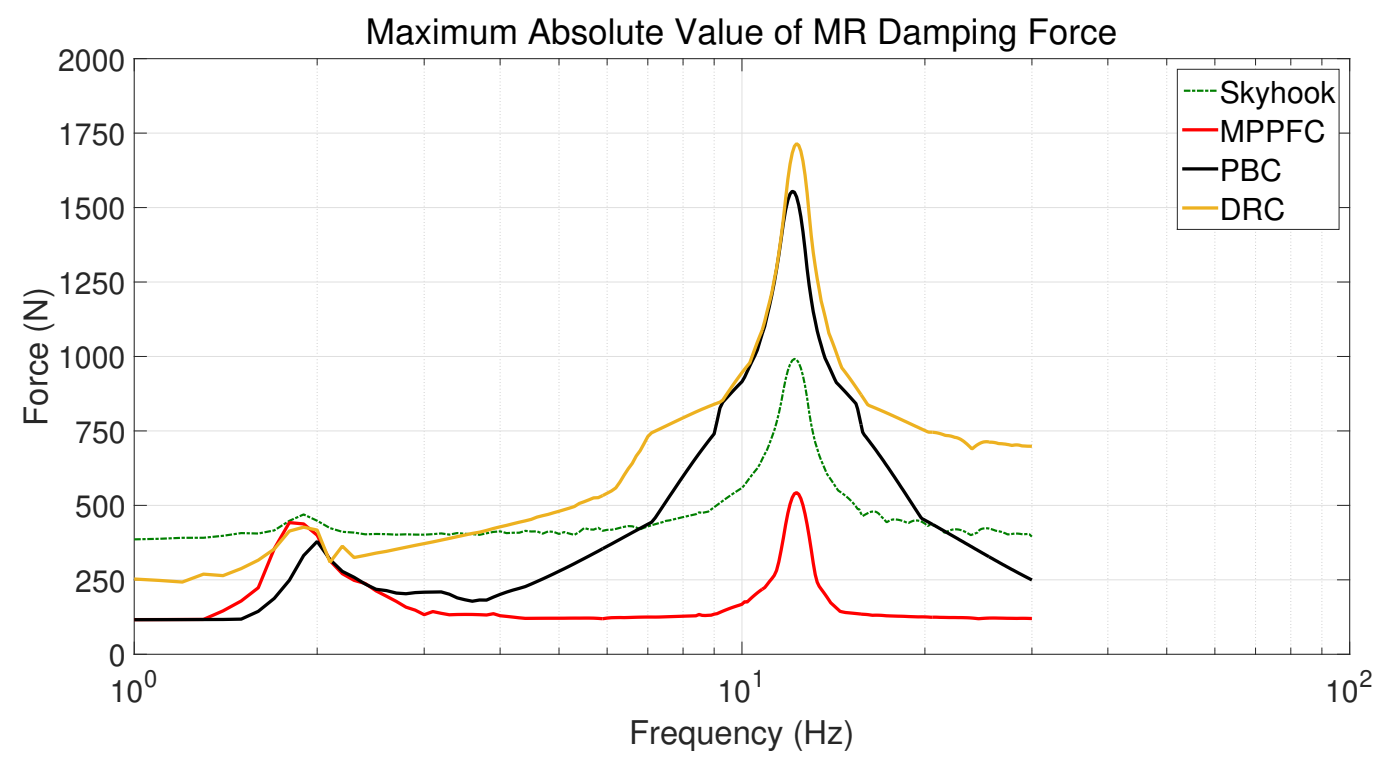

Figure 12. Damping force for the proposed semi-active control (SAC) schemes.

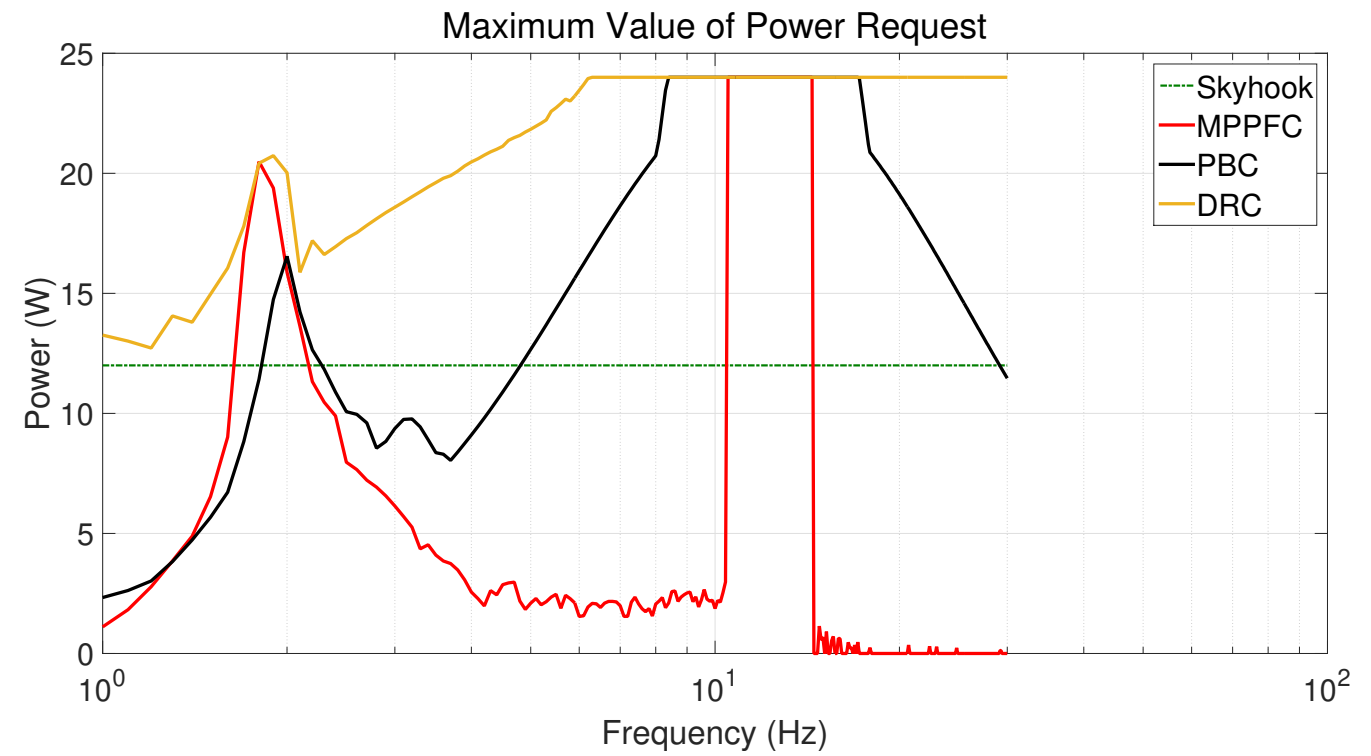

Figure 13. MRD power consumption for the proposed SAC schemes.

\section{Conclusions}

In this article, three semi-active modern control schemes for a quarter-vehicle suspension with magnetorheological damper (MRD) were developed to overcome the limitations of traditional semi-active control approaches and achieve the two main objectives with these kinds of systems, which are passenger comfort and the vehicle's tyre road holding. The proposed control schemes were quantitatively evaluated by performance indexes by following the methodology of analysis for automotive suspensions reported by Savaresi et al. [7], and compared with traditional sky-hook semi-active control, showing a considerable improvement in the closed-loop system performance at the two main vibration modes simultaneously. Furthermore, in order to carry out the numerical implementation of all proven controllers, the MR damper dynamics were taken into account by an inverse polynomial model for the current-damping force relationship. The proposed PBC and MPPFC had the simplest design and implementation procedure, therefore a low computing effort is requested, similar to classic SAC strategies. These characteristics are useful for real time automotive embedded systems. Although proposed DRC scheme had higher implementa- 
tion complexity than PBC and MPPFC, this strategy achieves the high robust and adaptive performance to reject endogenous and exogenous disturbances. In addition, synthesis and computing cost of proposed DRC scheme is even simpler than other modern SAC methods in the literature such as optimal hybrid or $H_{\infty}$ approaches. The obtained results show viability to experimentally implement the proposed SAC schemes in the laboratory test rig presented in [36] through the MRD considered in this work due to the obtained damping forces are within its operation range. This is the main future activity proposed to extend the presented numerical results. Regarding to quantified power consumption, $24 \mathrm{~W}$ is the maximum power provided to MRD which confirm the low power consumption for this kind of actuators. With this power, the MRD is able to generate damping forces up to $1700 \mathrm{~N}$. As can be observed in the obtained numerical results, the damping force depends on the particular SAC scheme and its maximum values are strongly related to the achieved vibration attenuation. This demonstrates the importance of considering other factors (related to hardware limitations) in the synthesis and performance evaluation of SAC schemes.

Author Contributions: Conceptualization, K.H.F.-A., M.A.-M. and Á.C.-A.; methodology, K.H.F.-A., M.A.-M., J.L.-F. and J.G.M.-L.; numerical simulation, K.H.F.-A., M.A.-M. and J.G.M.-L., validation, K.H.F.-A., M.A.-M. and J.G.M.-L.; resources, M.A.-M., J.L.-F., J.G.M.-L. and Á.C.-A.; writing—original draft preparation, K.H.F.-A. and M.A.-M.; writing - review and editing, M.A.-M., J.L.-F. and J.G.M.-L.; project administration, M.A.-M. All authors have read and agreed to the published version of the manuscript.

Funding: The authors received financial support from PRODEP-SEP, Mexico for publication of this article.

Institutional Review Board Statement: Not applicable.

Informed Consent Statement: Not applicable.

Conflicts of Interest: The authors declare no conflict of interest.

\section{References}

1. Ulasyar, A.; Lazoglu, I. Design and analysis of a new magneto rheological damper for washing machine. J. Mech. Sci. Technol. 2018, 32, 1549-1561. [CrossRef]

2. Spencer, B.F.; Dyke, S.J.; Sain, M.K.; Carlson, J.D. Phenomenological model for magnetorheological dampers. J. Eng. Mech. 1997, 123, 230-238. [CrossRef]

3. Aggumus, H.; Guclu, R. Robust $H_{\infty}$ control of STMDs used in structural systems by hardware in the loop simulation method. Actuators 2020, 9, 55. [CrossRef]

4. Rossi, A.; Orsini, F.; Scorza, A.; Botta, F.; Belfiore, N.P.; Sciuto, S.A. A review on parametric dynamic models of magnetorheological dampers and their characterization methods. Actuators 2018, 7, 16. [CrossRef]

5. Soon-Yong, Y.; Chulhee, H.; Sang-Un, S.; Seung-Bok, C. Design and evaluation of a semi-active magneto-rheological mount for a wheel loader cabin. Actuators 2017, 6, 16. [CrossRef]

6. Guglielmino, E.; Sireteanu, T.; Stammers, C.W.; Ghita, G.; Giuclea, M. Semi-Active Suspension Control: Improved Vehicle Ride and Road Friendliness; Springer: London, UK, 2008. [CrossRef]

7. Savaresi, S.M.; Poussot-Vassal, C.; Spelta, C.; Sename, O.; Dugard, L. Semi-Active Suspension Control Design for Vehicles; ButterworthHeinemann: Boston, MA, USA, 2010. [CrossRef]

8. Lozoya-Santos, J.J.; Morales-Menendez, R.; Ramirez-Mendoza, R.A. Control of an automotive semi-active suspension. Math. Probl. Eng. 2012, 2012, 218106. [CrossRef]

9. Sapinski, B.; Filus, J. Analysis of parametric models of MR linear damper. J. Theor. Appl. Mech. 2003, 41, 215-240.

10. Choi, S.B.; Lee, S.K.; Park, Y.P. A hysteresis model for the field dependent damping force of a magnetorheological damper. J. Sound Vib. 2001, 245, 375-383. [CrossRef]

11. Ortiz-Espinoza, A.A.; Cabello-Ortega, A.M.; Tudon-Martinez, J.C.; Hernandez-Alcantara, D.; Morales-Menendez, R. Analysis of on/off controllers of a semi-active suspension in a CAN. IFAC Proc. Vol. 2014, 47, 10902-10907. [CrossRef]

12. Brezas, P.; Smith, M.C.; Hoult, W. A clipped-optimal control algorithm for semi-active vehicle suspensions: Theory and experimental evaluation. Automatica 2015, 53, 188-194. [CrossRef]

13. Majdoub, K.E.; Giri, F.; Chaoui, F. Backstepping adaptive control of quarter-vehicle semi-active suspension with Dahl MR damper model. IFAC Proc. Vol. 2013, 46, 558-563. [CrossRef]

14. Felix-Herran, L.; Mehdi, D.; Ramirez-Mendoza, R.A.; Rodriguez-Ortiz, J.J.; Soto, R. $\mathrm{H}_{2}$ control of a one-quarter semi-active ground vehicle suspension. J. App. Res. Technol. 2016, 14, 173-183. [CrossRef] 
15. Mori, T.; Nilkhamhang, I.; Sano, A. Adaptive semi-active control of suspension system with MR damper. IFAC Proc. Vol. 2007, 40, 191-196. [CrossRef]

16. Cabrera-Amado, A.; Chavez-Conde, E.; Pablo-Altunar, J.M. Modelling and modal control of a quarter vehicle suspension system. In Modelacion Matematica: Ingenieria, Biologia y Ciencias Sociales; Universidad Tecnologica de la Mixteca: Oaxaca, Mexico, 2016; pp. 27-36. Available online: http://repositorio.utm.mx:8080/jspui/handle/123456789/156 (accessed on 28 December 2020).

17. Florean-Aquino, K.H.; Arias-Montiel, M.; Lugo-Gonzalez, E.; Cabrera-Amado, A. Single and multiple positive position feedback control of a magnetorheological automotive suspension. In Proceedings of the National Congress on Automatic Control, Puebla, Mexico, 23-25 October 2019; pp. 630-635.

18. Nguyen, M.; Sename, O.; Dugard, L. An LPV fault tolerant control for semi-active suspension-Scheduled by fault estimation. IFAC Pap. Online 2015, 48, 42-47. [CrossRef]

19. Han, J. From PID to active disturbance rejection control. IEEE Trans. Ind. Electron. 2009, 56, 900-906. [CrossRef]

20. Sun, L.; Liu, Y. Extended state observer augmented finite-time trajectory tracking control of uncertain mechanical systems. Mech. Syst. Signal. Process. 2020, 139, 106374. [CrossRef]

21. Han, S.; Wang, H.; Tian, Y. A linear discrete-time extended state observer-based intelligent PD controller for a 12 DOFs lower limb exoskeleton LLE-RePA. Mech. Syst. Signal. Process. 2020, 138, 106547. [CrossRef]

22. He, B.; Liu, G.; Chen, H.; Hu, X. Extended state observer-Based sliding mode learning control for mechanical system. Meas. Control 2020, 53, 1395-1403. [CrossRef]

23. Ren, C.; Ding, Y.; Ma, S. A structure-improved extended state observer based control with application to an omnidirectional mobile robot. ISA Trans. 2020, 101, 335-345. [CrossRef]

24. Arias-Montiel, M.; Florean-Aquino, K.H.; Francisco-Agustin, E.; Pinon-Lopez, D.M.; Santos-Ortiz, R.J.; Santiago-Marcial, B.A. Experimental characterization of a magnetorheological damper by a polynomial model. In Proceedings of the IEEE 2015 International Conference on Mechatronics, Electronics and Automotive Engineering (ICMEAE), Cuernavaca, Mexico, 24-27 November 2015; pp. 128-133. [CrossRef]

25. Goh, C.; Caughey, T. On the stability problem caused by finite actuator dynamics in the collocated control of large space structures. Int. J. Control 1985, 41, 787-802. [CrossRef]

26. Omidi, E.; Mahmoodi, S.N.; Shepard, W.S., Jr. Multi positive feedback control method for active vibration suppression in flexible structures. Mechatronics 2016, 33, 23-33. [CrossRef]

27. Cabrera-Amado, A.; Silva-Navarro, G. Semiactive vibration absorption in a rotor-bearing system using a ppf control scheme. In Proceedings of the International Conference on Noise and Vibration Engineering ISMA2012 + USD2012, Leuven, Belgium, 17-19 September 2012; pp. 209-221.

28. Inman, D.J. Vibration with Control; Wiley: Hoboken, NJ, USA, 2017. [CrossRef]

29. Ortega, R.; Loria, A.; Nicklasson, P.J.; Sira-Ramirez, H. Passivity-Based Control of Euler-Lagrange Systems; Springer: London, UK, 1998. [CrossRef]

30. Ortega, R.; van der Schaft, A.; Maschke, B.; Escobar, G. Interconnection and damping assignment passivity-based control of port-controlled Hamiltonian systems. Automatica 2002, 38, 585-596. [CrossRef]

31. Linares-Flores, J.; Reger, J.; Sira-Ramirez, H. Load torque estimation and passivity based control of a boost-converter/DC-motor combination. IEEE Trans. Control Syst. Technol. 2010, 18, 1398-1405. [CrossRef]

32. Sastry, S.S. Nonlinear Systems: Analysis, Stability, and Control; Springer: New York, NY, USA, 1999. [CrossRef]

33. Fliess, M.; Levine, J.; Martin, P.; Rouchon, P. Flatness and defect of nonlinear systems: Introductory theory and examples. Int. J. Control 1995, 61, 13-27. [CrossRef]

34. Sira-Ramirez, H.; Luviano-Juarez, A.; Ramirez-Neria, M.; Zurita-Bustamante, W. Active Disturbance Rejection Control of Dynamic Systems: A Flatness-Based Approach; Butterworth-Heinemann: Oxford, UK, 2017. [CrossRef]

35. Guerrero-Castellanos, J.; Rifai, H.; Arnez-Paniagua, V.; Linares-Flores, J.; Saynes-Torres, L.; Mohammed, S. Robust active disturbance rejection control via control Lyapunov functions: Application to actuated-ankle-foot-orthosis. Control Eng. Prac. 2018, 80, 49-60. [CrossRef]

36. Florean-Aquino, K.H.; Arias-Montiel, M.; Lugo-Gonzalez, E. Development of an experimental test rig of a semi-active suspension with magnetorheological damper. In Proceedings of the XXIII SOMIM Anual International Congress, Cuernavaca, Mexico, 20-22 September 2017; pp. 65-73. 